Published in "Theoretical Population Biology 106: 45-59, 2015"

which should be cited to refer to this work.

\title{
Is dispersal always beneficial to carrying capacity? New insights from the multi-patch logistic equation
}

\author{
Roger Arditi ${ }^{\mathrm{a}, \mathrm{d}, *}$, Claude Lobry ${ }^{\mathrm{b}, \mathrm{e}}$, Tewfik Sari ${ }^{\mathrm{c}, \mathrm{f}}$ \\ ${ }^{a}$ University of Fribourg, Department of Biology, Chemin du Musée 10, 1700 Fribourg, Switzerland \\ b INRA, INRIA, Projet Modemic, UMR Mistea, 2 place Pierre Viala, 34060 Montpellier Cedex 2, France \\ ' IRSTEA, UMR Itap, 361 rue Jean-François Breton, 34196 Montpellier Cedex 5, France \\ d Sorbonne Universités, UPMC Univ Paris 06, Institute of Ecology and Environmental Sciences (iEES-Paris), 75252 Paris Cedex 5, France \\ ${ }^{\mathrm{e}}$ Université de Nice-Sophia-Antipolis, France \\ ${ }^{\mathrm{f}}$ Université de Haute Alsace, LMIA, 4 rue des Frères Lumière, 68093 Mulhouse Cedex, France
}

\begin{abstract}
The standard model for the dynamics of a fragmented density-dependent population is built from several local logistic models coupled by migrations. First introduced in the 1970s and used in innumerable articles, this standard model applied to a two-patch situation has never been completely analysed. Here, we complete this analysis and we delineate the conditions under which fragmentation associated to dispersal is either beneficial or detrimental to total population abundance. Therefore, this is a contribution to the SLOSS question. Importantly, we also show that, depending on the underlying mechanism, there is no unique way to generalize the logistic model to a patchy situation. In many cases, the standard model is not the correct generalization. We analyse several alternative models and compare their predictions. Finally, we emphasize the shortcomings of the logistic model when written in the $r-K$ parameterization and we explain why Verhulst's original polynomial expression is to be preferred.
\end{abstract}

\section{Introduction}

The theoretical literature on spatially-distributed population dynamics is huge and we will make no attempt to review it. Instead, we will focus on some problems with the basic models that are used as the building blocks of this body of theory. Indeed, we have found that even the simplest and most ancient model still contained unresolved aspects and that unsupported generalizations were common. More precisely, we will explore the details of various ways to generalize the logistic model to a twopatch situation, i.e., the simplest way to describe the dynamics of a spatially-distributed, density-dependent population. The standard model commonly used in this situation has never been completely analysed. We will complete this analysis and we will delineate the conditions under which fragmentation can either be beneficial or detrimental to total population abundance. More importantly, we

\footnotetext{
* Corresponding author at: University of Fribourg, Department of Biology, Chemin du Musée 10, 1700 Fribourg, Switzerland.

E-mail addresses: roger.arditi@unifr.ch (R. Arditi), claude.lobry@inria.fr (C. Lobry), tewfik.sari@irstea.fr (T. Sari).
}

will show that this standard multi-patch logistic model is, in many cases, an incorrect description of the dynamics of a fragmented density-dependent population.

Assume that some population $N$ follows the logistic model when growing in a uniform environment:

$\frac{d N}{d t}=r N\left(1-\frac{N}{K}\right)$.

This model assumes perfect mixing of the population. For modelling the dynamics of the same species in a patchy environment, it is widely accepted to assume that each subpopulation in each patch follows a local logistic law and that the various patches are coupled by migrations. Taking the case of two patches as a simple example, the following model describes logistic growth in two patches linked symmetrically by migration:

$$
\left\{\begin{array}{l}
\frac{d N_{1}}{d t}=r_{1} N_{1}\left(1-\frac{N_{1}}{K_{1}}\right)+\beta\left(N_{2}-N_{1}\right), \\
\frac{d N_{2}}{d t}=r_{2} N_{2}\left(1-\frac{N_{2}}{K_{2}}\right)+\beta\left(N_{1}-N_{2}\right),
\end{array}\right.
$$

where $N_{i}$ is the population abundance in patch $i$ and $\beta N_{i}$ is the emigration flow from patch $i$ to the other patch $(\beta \geq 0)$. The 
parameters $r_{i}$ and $K_{i}$ are respectively the intrinsic growth rate and the carrying capacity in patch $i$. This model was first studied by Freedman and Waltman (1977), later by DeAngelis et al. (1979) and Holt (1985), and a graphical presentation was given by Hanski (1999, pp. 43-46) in his reference book on metapopulations. More recently, DeAngelis and Zhang (2014) have brought new developments.

We denote by $N_{1}^{*}$ and $N_{2}^{*}$ the population abundances at equilibrium. With no loss of generality, we assume that patch 1 has the lower carrying capacity (i.e., $\left.K_{1} \leq K_{2}\right)$. In isolation $(\beta=0)$, each population equilibrates at its local carrying capacity: $N_{i}^{*}=K_{i}$.

A well-known result is that, in the presence of dispersal $(\beta>0)$, the total equilibrium population, $N_{T}^{*}=N_{1}^{*}+N_{2}^{*}$, is generally different from the sum of the carrying capacities $K_{1}+K_{2}$. Freedman and Waltman (1977) have shown that, in the case of perfect mixing $(\beta \rightarrow \infty)$, both patch populations tend to equal values and that the total equilibrium population tends to:

$$
\begin{aligned}
N_{T}^{*}= & K_{1}+K_{2} \\
& +\left(K_{1}-K_{2}\right) \frac{r_{1} K_{2}-r_{2} K_{1}}{r_{1} K_{2}+r_{2} K_{1}}, \quad \text { in the limit } \beta \rightarrow \infty .
\end{aligned}
$$

(Note that this expression contained typos in Freedman and Waltman, 1977, their equation 3.3 that were only partially corrected by Holt, 1985.)

Depending on the sign of the numerator present in Eq. (3), dispersal can either be beneficial or detrimental with respect to the total carrying capacity. Thus, if $r_{1} K_{2}<r_{2} K_{1}$ (with $K_{1}<K_{2}$ ), we will have

$N_{T}^{*}>K_{1}+K_{2}, \quad$ if $\beta$ is sufficiently large.

This spectacular result, somewhat paradoxical, has been widely discussed and has led to speculations about the general virtues of patchiness and dispersal, for example in the context of the conservation ecology question of whether a single large refuge is better or worse than several small ones (the SLOSS debate; see, e.g., Hanski, 1999).

Freedman and Waltman (1977) only contrasted the situations of perfect isolation and perfect mixing; they did not study the effect of intermediate values of the dispersal parameter $\beta$. This effect was studied in the recent paper of DeAngelis and Zhang (2014), but only in the special case $r_{1} / K_{1}=r_{2} / K_{2}$.

In the present paper, we will bring two contributions. Firstly, in Section 2 and Appendix A, we will present the analysis of model (2) in the full parameter space. We will show how the effects of dispersal $\beta$ and of the $r_{i} / K_{i}$ ratios combine and we will determine the exact conditions under which $N_{T}^{*}>K_{1}+K_{2}$ (see Proposition 2). These results have importance in those cases in which model (2) is a relevant description of logistic growth in a patchy environment.

Our second contribution will be to question the general validity of system (2) for modelling a patchy logistic population, using several simple examples. The logistic model is often justified on phenomenological grounds. However, it can also be derived from mechanistic considerations. Depending on the mechanism being considered, we will show that the correct generalization to a patchy situation is not necessarily represented by model (2) and that the equilibrium total population can be different from that predicted by this model. More precisely, we will show in Section 3 (with Appendix B) and in Section 4 (with Appendix C) that the patch coupling (2) is incorrect in models in which logistic growth is due to resource exploitation, while it is correct in a model in which logistic growth arises from agonistic inter-individual interactions (see Section 5).

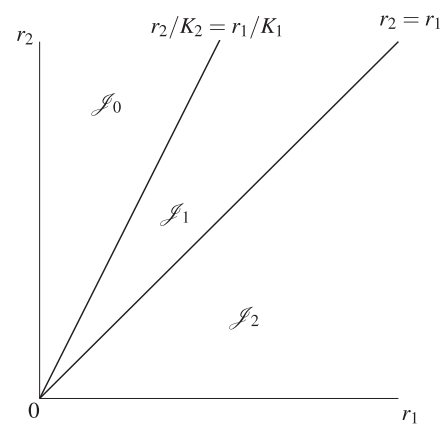

Fig. 1. Qualitative properties of model (2). In $\mathscr{J}_{0}$, patchiness has a beneficial effect on total carrying capacity. This effect is detrimental in $\mathscr{J}_{2}$. In $\mathscr{J}_{1}$, the effect is beneficial for lower values of the migration coefficient $\beta$ and detrimental for the higher values. Note that, because of the assumption $K_{1} \leq K_{2}$, the two oblique lines cannot be reversed. See text in Section 2 for additional explanations.

\section{Theoretical analysis of the standard two-patch logistic model}

In this section, we summarize some of the properties of the standard model (2). Formal proofs are given in the Mathematical Appendix A.

As already mentioned in the Introduction, with no dispersal $(\beta=0)$, each patch equilibrates at its own carrying capacity and the total equilibrium number of individuals is just the sum of the carrying capacities: $N_{T}^{*}=K_{1}+K_{2}$. This remains true with dispersal $(\beta>0)$ if the two carrying capacities are identical. However, if the carrying capacities are not identical $\left(K_{1}<K_{2}\right)$, the equilibrium densities are such that

$K_{1}<N_{1}^{*}<N_{2}^{*}<K_{2}$,

meaning that, in general, $N_{T}^{*} \neq K_{1}+K_{2}$ (see Proposition 2 in Appendix A).

In particular, the total equilibrium population $N_{T}^{*}$ can be greater than the sum of the carrying capacities. In the Introduction, we mentioned Freedman and Waltman's result in the case of perfect mixing $(\beta \rightarrow \infty)$ (Eqs. (3)-(4)). This can also occur with imperfect mixing as, for example, if $r_{1} / K_{1}<r_{2} / K_{2}$ (with $K_{1}<K_{2}$ ). In this case, as shown in Appendix A,

$N_{T}^{*}>K_{1}+K_{2}, \quad$ as soon as $\beta>0$.

Note that, if migration is asymmetric $\left(\beta_{1} \neq \beta_{2}\right)$, then it is possible to have $N_{T}^{*}>K_{1}+K_{2}$ even in the case $K_{1}=K_{2}$ (Poggiale et al., 2005).

Appendix A gives the full mathematical analysis of the equilibrium properties of the coupled logistic model (2). The main qualitative results are summarized by Fig. 1. Depending on the inequalities between $r_{1}$ and $r_{2}$, and between $r_{1} / K_{1}$ and $r_{2} / K_{2}$, three different domains must be considered in the parameter space $r_{1} \times r_{2}$. We define $\mathscr{J}_{0}$ by the condition $r_{2} / K_{2} \geq r_{1} / K_{1}, \mathscr{J}_{2}$ by the condition $r_{2} \leq r_{1}$, and $\mathscr{J}_{1}$ by the condition $r_{2} / K_{2}<r_{1} / K_{1}$ and $r_{2}>r_{1}$.

The effect of patchiness and migration is different in the three domains. In $\mathscr{J}_{0}$, this effect is beneficial: $N_{T}^{*}$ is always greater than $K_{1}+K_{2}$. In $\mathscr{J}_{2}$, the opposite is true: patchiness is detrimental since $N_{T}^{*}$ is always smaller than $K_{1}+K_{2}$. In $\mathscr{J}_{1}$, the effect of patchiness depends on the migration rate: it is beneficial at lower values of the migration coefficient $\beta$ while this effect becomes detrimental at high values. This is illustrated by Fig. 2, in which the total equilibrium abundance $N_{T}^{*}$ is plotted as a function of the migration rate $\beta$. Depending on the choice of parameter values (given in Table 1), this figure shows three different example patterns, belonging respectively to $\mathscr{J}_{0}, \mathscr{J}_{2}$, and $\mathscr{J}_{1}$.

Fig. 2(a) is an example response in $\mathscr{J}_{0}$ : as soon as there is some migration $(\beta>0)$, the global carrying capacity $N_{T}^{*}$ is greater than $K_{1}+K_{2}$. In Fig. 2(b), we show an example response in $\mathscr{J}_{2}$ : the total 

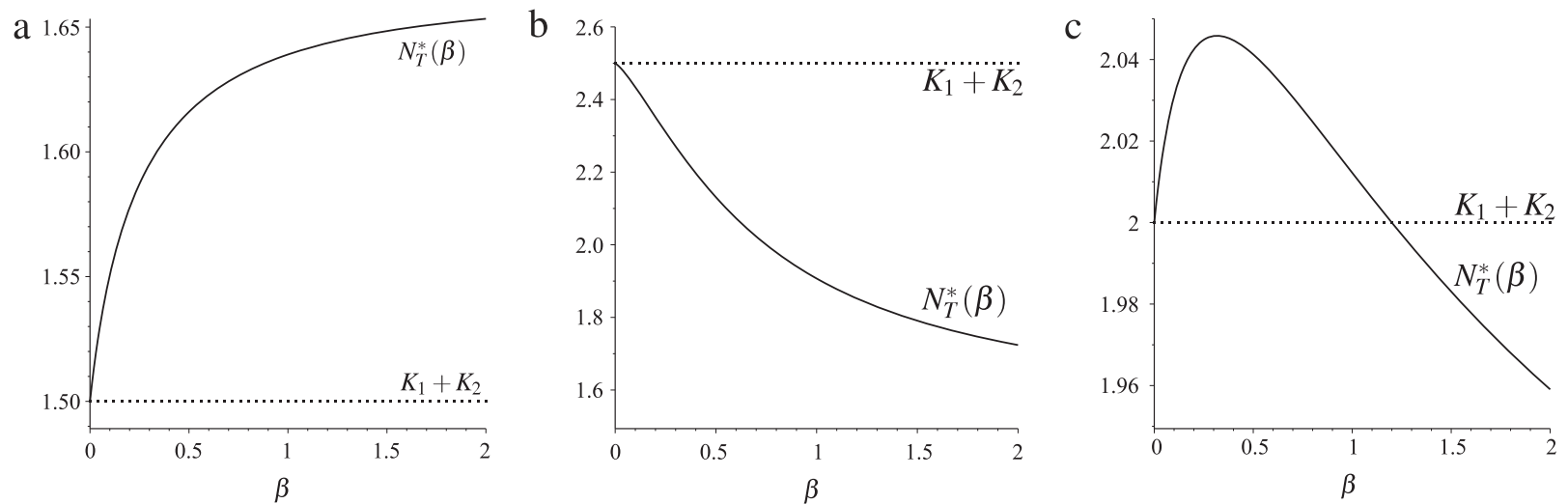

Fig. 2. Total equilibrium population of model (2) as a function of migration: $N_{T}^{*}(\beta)$. The horizontal dotted line is $K_{1}+K_{2}$. Depending on the parameter values (given in Table 1), three different patterns can be obtained, corresponding to the three domains of Fig. 1. (a) Example in $\mathscr{J}_{0}$. (b) Example in $\mathscr{J}_{2}$. (c) Example in $\mathscr{J}_{1}$. See text in Section 2 for more comments.

\section{Table 1}

Parameter values of the three cases of Fig. 2. The derivative $\frac{d N_{T}^{*}}{d \beta}(0)$ is calculated with the expression given in item 1 of Proposition 4, and the perfect mixing abundance $N_{T}^{*}(+\infty)$ with Eq. (A.13).

\begin{tabular}{lllllll}
\hline & $r_{1}$ & $K_{1}$ & $r_{2}$ & $K_{2}$ & $\frac{d N_{T}^{*}}{d \beta}(0)$ & $N_{T}^{*}(+\infty)$ \\
\hline Fig. 2(a) & 0.5 & 0.5 & 2 & 1 & $0.75>0$ & $1.67>K_{1}+K_{2}$ \\
Fig. 2(b) & 1 & 0.5 & 0.8 & 2 & $-0.375<0$ & $1.5<K_{1}+K_{2}$ \\
Fig. 2(c) & 1 & 0.5 & 2 & 1.5 & $0.5>0$ & $1.8<K_{1}+K_{2}$
\end{tabular}

equilibrium population $N_{T}^{*}$ is always lower than $K_{1}+K_{2}$. Finally, Fig. 2(c) shows a response in the intermediate domain $\mathscr{J}_{1}$, in which the lower values of the migration rate have a beneficial effect while this effect becomes detrimental at high values.

\section{Mechanism 1: Logistic growth induced by resource con- sumption}

Having given in Section 2 the full analysis of the two-patch logistic model (2), we now turn to the second contribution of this paper, i.e., the correct way to build patch models derived from mechanistic considerations. For the first mechanism, we take the example of a population of bacteria consuming a substrate in a batch culture. This process occurs on a fast time scale, on which bacterial mortality can be ignored. Assuming perfect mixing of both the substrate and the population, this situation is modelled by:

$$
\left\{\begin{array}{l}
\frac{d R}{d t}=-a R N \\
\frac{d N}{d t}=\varepsilon a R N
\end{array}\right.
$$

where $R$ is the substrate concentration, $N$ the bacterial density, $a$ the so-called "searching efficiency" of the mass-action interaction, and $\varepsilon$ the conversion coefficient.

We have:

$\frac{d(\varepsilon R+N)}{d t}=0$

and thus $\varepsilon R(t)+N(t)=\varepsilon R(0)+N(0)=M$. Substituting $(M-N)$ to $\varepsilon R$ in the second equation of (7), one gets:

$$
\frac{d N}{d t}=a N(M-N),
$$

which is equivalent to the logistic equation (1) with $K=M$ and $r=a M$. This equation, derived from (7), has long ago been shown to give an excellent empirical description of the dynamics of a batch culture of micro-organisms (e.g., Pearl, 1927).
Consider now two coupled batch reactors, with the same bacteria and substrate, and differing only in the initial conditions. Denote by $R_{1}, N_{1}, R_{2}$, and $N_{2}$ the population sizes of substrate and bacteria in reactors 1 and 2 respectively. Let $M_{1}=\varepsilon R_{1}(0)+N_{1}(0)$ and $M_{2}=\varepsilon R_{2}(0)+N_{2}(0)$. If we assume linear dispersal between the two patches and if we ignore the consumption mechanism that led to Eq. (8), it is tempting to model the coupled reactors by coupling the corresponding reduced Eqs. (8) with the addition of migrations:

$\left\{\begin{array}{l}\frac{d N_{1}}{d t}=a N_{1}\left(M_{1}-N_{1}\right)+\beta\left(N_{2}-N_{1}\right) \\ \frac{d N_{2}}{d t}=a N_{2}\left(M_{2}-N_{2}\right)+\beta\left(N_{1}-N_{2}\right)\end{array}\right.$

We said in the previous section (and proved in the Mathematical Appendix A) that, if we denote by $\left(N_{1}^{*}, N_{2}^{*}\right)$ the equilibrium of (9), then

$N_{1}^{*}+N_{2}^{*}>M_{1}+M_{2}$

as soon as $\beta>0$ and $M_{1} \neq M_{2}$.

Despite its perfect mathematical derivation, this result is false for the coupling of the two reactors. The correct description in this case must be done by modelling the consumption mechanism (7) in the two patches with possibly different dispersal rates for the substrate $(\alpha)$ and for the bacteria $(\beta)$ :

$\left\{\begin{array}{l}\frac{d R_{1}}{d t}=-a R_{1} N_{1}+\alpha\left(R_{2}-R_{1}\right), \\ \frac{d R_{2}}{d t}=-a R_{2} N_{2}+\alpha\left(R_{1}-R_{2}\right), \\ \frac{d N_{1}}{d t}=\varepsilon a R_{1} N_{1}+\beta\left(N_{2}-N_{1}\right), \\ \frac{d N_{2}}{d t}=\varepsilon a R_{2} N_{2}+\beta\left(N_{1}-N_{2}\right) .\end{array}\right.$

Adding the four equations, one gets:

$\frac{d\left(\varepsilon R_{1}+\varepsilon R_{2}+N_{1}+N_{2}\right)}{d t}=0$,

which means:

$$
\begin{aligned}
& \varepsilon R_{1}(t)+\varepsilon R_{2}(t)+N_{1}(t)+N_{2}(t) \\
& \quad=\varepsilon R_{1}(0)+\varepsilon R_{2}(0)+N_{1}(0)+N_{2}(0)=M_{1}+M_{2} .
\end{aligned}
$$

Therefore, we always have, including at equilibrium:

$N_{1}(t)+N_{2}(t) \leq M_{1}+M_{2}$

because the quantity $R_{1}(t)+R_{2}(t)$ must be positive! 
Since at equilibrium $R_{1}^{*}=R_{2}^{*}=0$, we conclude that

$N_{1}^{*}+N_{2}^{*}=M_{1}+M_{2}$,

in contradiction with the result (10) obtained when coupling the reduced logistic models (8). This is completely independent of the value of $\alpha$, the substrate dispersal rate.

The flaw in obtaining the wrong inequality (10) was that it was derived on the basis of the reduced model (9), itself derived from the first integral $\varepsilon R(t)+N(t)=\varepsilon R(0)+N(0)$ of $(7)$, which is no longer a first integral of the full system (11).

\section{Mechanism 2: Logistic growth induced by MacArthur's reduction}

The second mechanistic derivation of logistic growth that we consider is that of MacArthur (1969) and MacArthur (1970). ${ }^{1}$ Consider the following model for resource-consumer dynamics:

$\left\{\begin{array}{l}\frac{d R}{d t}=\left[s\left(1-\frac{R}{L}\right)-a N\right] R, \\ \frac{d N}{d t}=\varepsilon(a w R-q) N,\end{array}\right.$

where $R$ is the population density of the resource (prey), $N$ is the population density of the consumer (predator), $a$ is the searching efficiency (as in Section 3), $w$ is the weight (caloric value) of the resource, $q$ is the metabolic rate for maintenance of the consumer, and $\varepsilon$ is a proportionality constant governing the biochemical conversion of resource $R$ into consumer $N$. The resource is assumed to follow logistic growth with parameters $s$ and $L$ when the consumer is not present. For the sake of simplicity, we denote $b=a w$.

The system (12) is a standard model built for a biotic resource with logistic intrinsic dynamics and a consumer with LotkaVolterra functional response. It has been used in a very large number of articles that generalized it to multi-patch and/or to multi-species situations. The model is more appropriate than the mechanism of Section 3 when the resource can reproduce with its own dynamics and when the consumer has some loss term (e.g., due to basal metabolism or mortality).

MacArthur's contribution was to make the crucial assumption that the conversion coefficient $\varepsilon$ was small. Taking advantage of the separation of time scales, the "quasi-steady state" of the resource can be calculated from the (fast) first equation in (12) and be used to replace $R$ in the second equation. The quasi-steady state $R$, that is, the solution of the algebraic equation $s(1-R / L)-a N=0$, is given by

$R=L\left(1-\frac{a}{s} N\right)$

and substituting it into the second equation in (12) gives:

$\frac{d N}{d t}=\varepsilon\left(b L-q-\frac{a b}{s} L N\right) N$,

which is once more the logistic equation (1) with intrinsic growth rate and carrying capacity

$r=\varepsilon(b L-q), \quad K=\frac{s}{a} \frac{b L-q}{b L}$.

Note that the above reduction method was recently generalized to other types of resource-consumer systems by Reynolds and Brassil (2013).

\footnotetext{
1 Anecdotally, this was the very first article published in Theoretical Population Biology (vol. 1, issue 1, page 1).
}

Let us now consider two patches and assume some migration between the two. If we model this situation directly with MacArthur's reduced logistic form (13), we have:

$$
\left\{\begin{array}{l}
\frac{d N_{1}}{d t}=\varepsilon_{1}\left(b_{1} L_{1}-q_{1}-\frac{a_{1} b_{1}}{s_{1}} L_{1} N_{1}\right) N_{1}+\beta\left(N_{2}-N_{1}\right), \\
\frac{d N_{2}}{d t}=\varepsilon_{2}\left(b_{2} L_{2}-q_{2}-\frac{a_{2} b_{2}}{s_{2}} L_{2} N_{2}\right) N_{2}+\beta\left(N_{1}-N_{2}\right) .
\end{array}\right.
$$

The population in each patch follows logistic growth with intrinsic growth rates

$r_{1}=\varepsilon_{1}\left(b_{1} L_{1}-q_{1}\right), \quad r_{2}=\varepsilon_{2}\left(b_{2} L_{2}-q_{2}\right)$,

and carrying capacities

$K_{1}=\frac{s_{1}}{a_{1}} \frac{b_{1} L_{1}-q_{1}}{b_{1} L_{1}}, \quad K_{2}=\frac{s_{2}}{a_{2}} \frac{b_{2} L_{2}-q_{2}}{b_{2} L_{2}}$.

With no migration ( $\beta=0$ ), each patch equilibrates at its respective carrying capacity and the total number of individuals present at equilibrium is just the sum of the carrying capacities, $N_{T}^{*}$ $=K_{1}+K_{2}$.

Let us denote by $\left(N_{1}^{*}, N_{2}^{*}\right)$ the positive (and globally stable) equilibrium of (14). We will compare the total population $N_{T}^{*}=$ $N_{1}^{*}+N_{2}^{*}$ with the total population obtained for the complete twopatch extension of (12), which is:

$\left\{\begin{array}{l}\frac{d R_{1}}{d t}=\left[s_{1}\left(1-\frac{R_{1}}{L_{1}}\right)-a_{1} N_{1}\right] R_{1}+\alpha\left(R_{2}-R_{1}\right), \\ \frac{d R_{2}}{d t}=\left[s_{2}\left(1-\frac{R_{2}}{L_{2}}\right)-a_{2} N_{2}\right] R_{2}+\alpha\left(R_{1}-R_{2}\right), \\ \frac{d N_{1}}{d t}=\varepsilon_{1}\left(b_{1} R_{1}-q_{1}\right) N_{1}+\beta\left(N_{2}-N_{1}\right), \\ \frac{d N_{2}}{d t}=\varepsilon_{2}\left(b_{2} R_{2}-q_{2}\right) N_{2}+\beta\left(N_{1}-N_{2}\right) .\end{array}\right.$

The term $\alpha\left(R_{2}-R_{1}\right)$ represents some possible migration of the resource, which is not present in the reduced system (14) since the variable $R$ does not appear in the equations.

Computer simulations show that system (17) has an equilibrium, which appears to be globally stable, and we denote it by

$E^{* *}=\left(R_{1}^{* *}, R_{2}^{* *}, N_{1}^{* *}, N_{2}^{* *}\right)$.

We will now compare the effect of migrations expressed in the complete model (17) and in the reduced model (14). This will be done by simulation. We integrate the equations for a long time (namely 100 units of time) until the equilibrium is almost reached and then compute the total consumer population for both models (14) and (17):

$N_{T}^{*}=N_{1}^{*}+N_{2}^{*}, \quad N_{T}^{* *}=N_{1}^{* *}+N_{2}^{* *}$.

Although the general case of different migration rates $\alpha$ and $\beta$ can be studied with no special difficulty, we will consider in this paper two special cases in order to simplify the presentation:

$\alpha=\beta \geq 0, \quad$ in Section 4.1,

and

$\alpha=0$ and $\beta \geq 0, \quad$ in Section 4.2.

In order to single out the role of migration strength, we will compare the graphs of $N_{T}^{*}(\beta)$ and $N_{T}^{* *}(\beta)$ as functions of $\beta$, all other parameters being fixed. 
Table 2

Numerical values of the parameters of model (17) with $\alpha=\beta$ used in Figs. 3 and 4 .

\begin{tabular}{lllllllllllll}
\hline & $s_{1}$ & $L_{1}$ & $q_{1}$ & $s_{2}$ & $L_{2}$ & $q_{2}$ & $a_{1}$ & $a_{2}$ & $b_{1}$ & $b_{2}$ & $\varepsilon_{1}$ & $\varepsilon_{2}$ \\
\hline Fig. 3(a) & 1 & 1 & 0.5 & 3 & 3 & 2 & 1 & 1 & 1 & 1 & 1 & 1 \\
Fig. 3(b) & 1 & 2.8 & 1.5 & 3 & 3.2 & 0.5 & 1 & 1 & 1 & 1 & 1 & 1 \\
Fig. 3(c) & 1 & 2 & 0.5 & 3 & 2 & 2 & 1 & 0.8 & 1 & 2 & 1 & 0.1 \\
Fig. 3(d) & 1 & 1.5 & 1 & 1 & 3 & 2 & 1 & 0.5 & 1 & 1.5 & 0.5 & 0.5 \\
Fig. 4 & 1 & 1 & 0.1 & 1 & 2.5 & 1 & 4 & 0.1 & 1 & 1 & 0.6 & 0.4 \\
\hline
\end{tabular}

Table 3

Numerical values of several quantities derived from the parameters of Table 2. $r_{1}$, $r_{2}, K_{1}, K_{2}$ are calculated with Eqs. (15)-(16). The derivatives $\frac{d N_{T}^{*}}{d \beta}(0)$ and $\frac{d N_{T}^{* *}}{d \beta}(0)$, and the perfect mixing abundances $N_{T}^{*}(\infty)$ and $N_{T}^{* *}(\infty)$ are calculated with the expressions given in Eqs. (B.2), (B.5), and (B.6).

\begin{tabular}{lllllccll}
\hline & $r_{1}$ & $r_{2}$ & $K_{1}$ & $K_{2}$ & $\frac{d N_{T}^{*}}{d \beta}(0)$ & $\frac{d N_{T}^{* *}}{d \beta}(0)$ & $N_{T}^{*}(\infty)$ & $N_{T}^{* *}(\infty)$ \\
\hline Fig. 3(a) & 0.5 & 1 & 0.5 & 1 & 0.5 & 2.75 & 1.5 & 1.5 \\
Fig. 3(b) & 1.3 & 2.7 & 0.464 & 2.531 & 0.824 & 2.158 & 2.069 & 2.705 \\
Fig. 3(c) & 1.5 & 0.2 & 0.75 & 1.875 & -4.875 & -4.5 & 1.614 & 3.148 \\
Fig. 3(d) & 0.25 & 1.25 & 0.333 & 1.111 & 2.489 & 2.322 & 1.6 & 1.067 \\
Fig. 4 & 0.54 & 0.6 & 0.225 & 6 & 1.07 & -5.68 & 0.91 & 0.66 \\
\hline
\end{tabular}

\subsection{Migration of both the resource and the consumer}

In this section, the migration between the two patches is $\alpha=\beta$ with $\beta \geq 0$. The simulations will be done with the values given in Table 2 . The parameters leading to the example behaviours of Figs. 3 and 4 are chosen in such way that the population derivatives at $\beta=0$ and the population values at $\beta=\infty$ obey a variety of inequalities. In other words, the parameters shown in Table 2 are chosen in such way that the quantities shown in the last four columns of Table 3 verify typical inequalities. This was done with help of Propositions 5 and 6, which are presented in Appendix B.

In Figs. 3 and 4 , the value of $K_{1}+K_{2}$ is represented by the horizontal dotted line. As soon as $\beta$ is strictly positive, there is a departure from this value and the pictures also show that the values predicted by the reduced model are quite different from those predicted by the complete model. This is not surprising since the reduced model does not take into account the resource migration modelled by $\alpha=\beta>0$.

We first set the parameter values as in line 1 of Table 2 . In this example (Fig. 3(a)), the total population with migrations for the complete model is always greater than the total population with migrations for the reduced model, which is itself always greater than the total population without migration. This is not true in general, as shown by Fig. 3(b), obtained with the parameter values in line 2 of Table 2 . In this example, $K_{1}+K_{2} \approx 2.995$ (see line 2 of Table 3 ). We see that small migration values improve the total population but large values deteriorate it. Therefore the total population is not always greater than the sum of the two carrying capacities, nor the total population for the complete model is always greater than the total population for the reduced model. More precisely, all the possible inequalities between $N_{T}^{*}, N_{T}^{* *}$, and $K_{1}+K_{2}$ can actually be obtained, depending on the value of the migration intensity $\beta$, as shown by Fig. 3(b), in which we have successively: $N_{T}^{* *}>N_{T}^{*}>K_{1}+K_{2}, N_{T}^{*}>N_{T}^{* *}>K_{1}+K_{2}$, $N_{T}^{* *}>K_{1}+K_{2}>N_{T}^{*}, K_{1}+K_{2}>N_{T}^{*}>N_{T}^{* *}$, and $K_{1}+K_{2}>N_{T}^{* *}>N_{T}^{*}$.

Fig. 3(c) is obtained with the parameter values in line 3 of Table 2. We see in Table 3 that, for these parameter values, we have $r_{2}<r_{1}$. Therefore, from the theoretical results of Section 2 (see Fig. 1), we deduce that $N_{T}^{*}(\beta)<K_{1}+K_{2}$ for any $\beta>0$ and $N_{T}^{*}(\beta)$ is decreasing, as illustrated in Fig. 3(c). However, we see on this figure that $N_{T}^{* *}(\beta)$ decreases first and then increases, and can take values larger than $K_{1}+K_{2}$.

Fig. 3(d) is obtained with the parameter values in line 4 of Table 2. We see in Table 3 that, for these parameter values, we have $r_{2} / K_{2}>r_{1} / K_{1}$. Therefore, from Fig. 1 , we deduce that $N_{T}^{*}(\beta)>$
Table 4

Numerical values of the parameters of model (17) with $\alpha=0$ used in Figs. 5 and C.8. The values of the other parameters in (17) are $\varepsilon_{i}=a_{i}=b_{i}=1$.

\begin{tabular}{lllllllllll}
\hline & $s_{1}$ & $L_{1}$ & $q_{1}$ & $s_{2}$ & $L_{2}$ & $q_{2}$ & $r_{1}$ & $K_{1}$ & $r_{2}$ & $K_{2}$ \\
\hline Fig. 5(a) & 3 & 3 & 2.5 & 2.5 & 2.5 & 0.2 & 0.5 & 0.5 & 2.3 & 2.3 \\
Fig. 5(b) & 1 & 1 & 0.4 & 3 & 3 & 1 & 0.6 & 0.6 & 2 & 2 \\
\hline
\end{tabular}

$K_{1}+K_{2}$ for any $\beta>0$, as illustrated in Fig. 3(d). However, we see on this figure that $N_{T}^{* *}(\beta)>K_{1}+K_{2}$ for small $\beta$ and the opposite holds for large $\beta$.

Fig. 4 is obtained with the parameter values in line 5 of Table 2. We see in Table 3 that, for these parameter values, we have $r_{2}>r_{1}$ and $r_{2} / K_{2}<r_{1} / K_{1}$. Therefore, from Fig. 1, we have that $N_{T}^{*}(\beta)>$ $K_{1}+K_{2}$ for $\beta$ small enough and $N_{T}^{*}(\beta)<K_{1}+K_{2}$ for $\beta$ large enough, as illustrated in Fig. 4. However, we see on this figure that $N_{T}^{* *}(\beta)$ has a completely different behaviour. It should be noticed (see Table 3) that $\frac{d N_{T}^{* *}}{d \beta}(0)<0$ and $\frac{d N_{T}^{*}}{d \beta}(0)>0$, as also shown in the zoom in Fig. 4(b). Hence, $N_{T}^{* *}(\beta)$ is first decreasing, then increasing, then decreasing again, while $N_{T}^{*}(\beta)$ is first increasing and then decreasing.

\subsection{Migration of the consumer alone}

Since MacArthur's reduction does not contain the resource as an explicit variable, it certainly cannot, as shown above, account for resource migration. However, if we assume that there is no resource migration, one may wonder whether it does not become accurate. The complete model is system (17) with $\alpha=0$. It can be analysed mathematically to a large extent (see Appendix C). Here, we present illustrations obtained by numerical simulation.

We set the parameter values as in line 1 of Table 4. In Fig. 5(a), the value $K_{1}+K_{2}$ is represented by the horizontal dotted line. As soon as $\beta$ is strictly positive, there is a departure from this value. The value predicted by the reduced model is the same as the one predicted by the complete model. Indeed, for this set of parameters, the reduced model gives a correct picture of the complete model. However, this is not the general case, as will be shown in the following example.

We now set the parameters as in line 2 of Table 4. In Fig. 5(b), the value $K_{1}+K_{2}$ is again represented by the horizontal dotted line. As soon as $\beta$ is strictly positive, there is a departure from this value. The value predicted by the reduced model is the same as the one predicted by the complete model when $\beta$ is small enough. After a certain value ( $\beta \approx 0.5$ ), the predictions of the two models differ suddenly, with the prediction of the reduced model (solid curve) being quite smaller than the prediction of the complete model (curve with circles). The mathematical explanation to this threshold effect is given in Appendix C.

Regarding the abundances, the stable equilibrium (18) is strictly positive when $\beta<0.5$, but when $\beta>0.5$, the resource $R_{1}$ becomes extinct at equilibrium, as shown in Fig. 6 . Thus, when $\beta>0.5$, the system works as a classical source-sink system, with the resource being constantly supplied by patch 2 to patch 1 , where it is instantly consumed by the population $N_{1}$.

\section{Discussion}

When we say that the logistic equation (1) is a model for the growth of some population $N$, what do we mean exactly? Usually, we say nothing about the actual mechanisms that explain this kind of growth. What we mean is roughly the following argument:

1. Let $\mu(N)$ be the density-dependent, specific growth rate of a population. If we want the population to be bounded, $\mu(N)$ must decrease to 0 . 

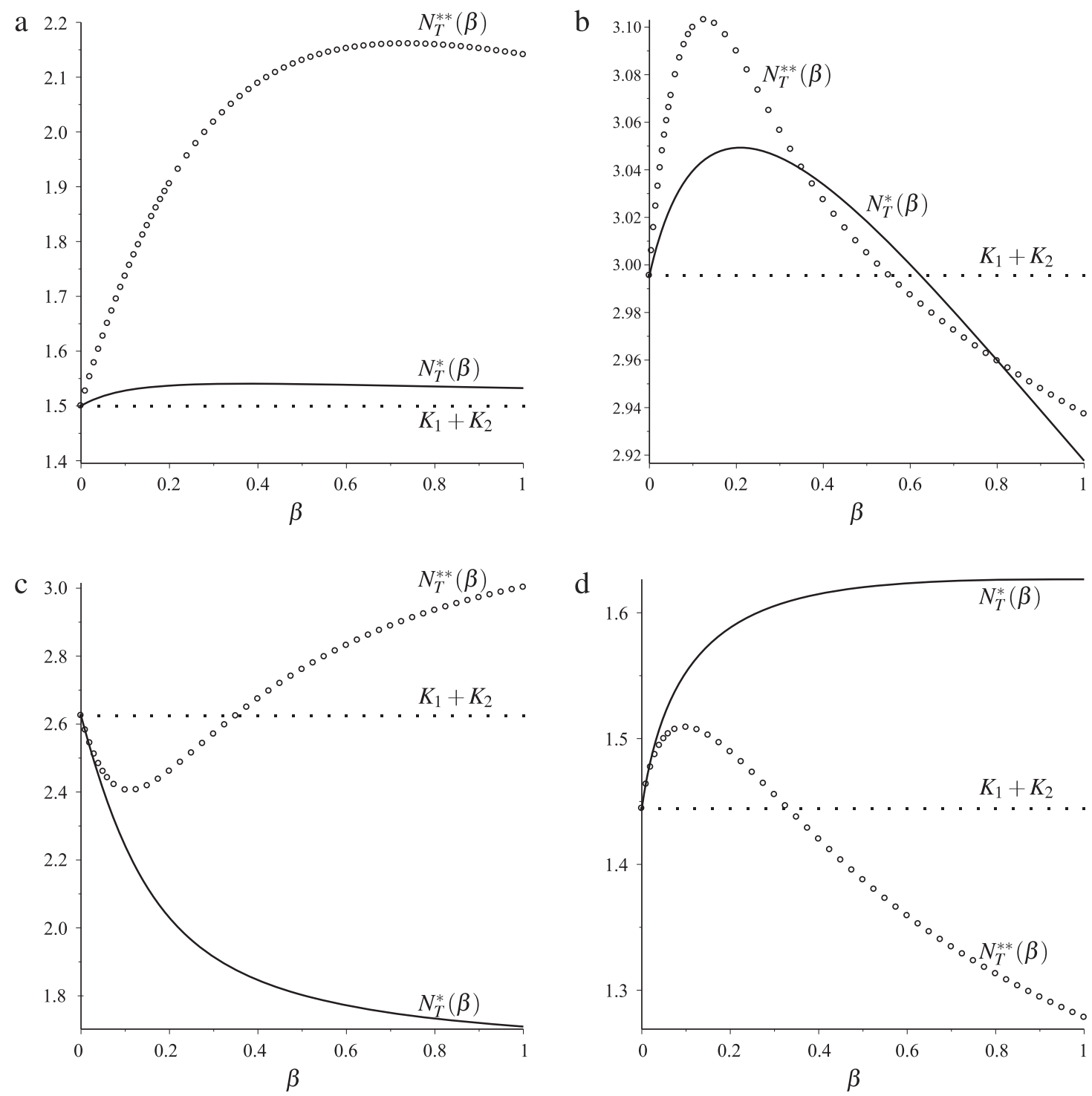

Fig. 3. Total consumer population as a function of migration when both the resource and the consumer can disperse. $N_{T}^{*}$ (solid curve) for the coupled-logistic reduced model, $N_{T}^{* *}$ (curve with circles) for the complete mechanistic model. See Tables 2 and 3 for the parameter values and text in Section 4.1 for explanations. See also Fig. 4.
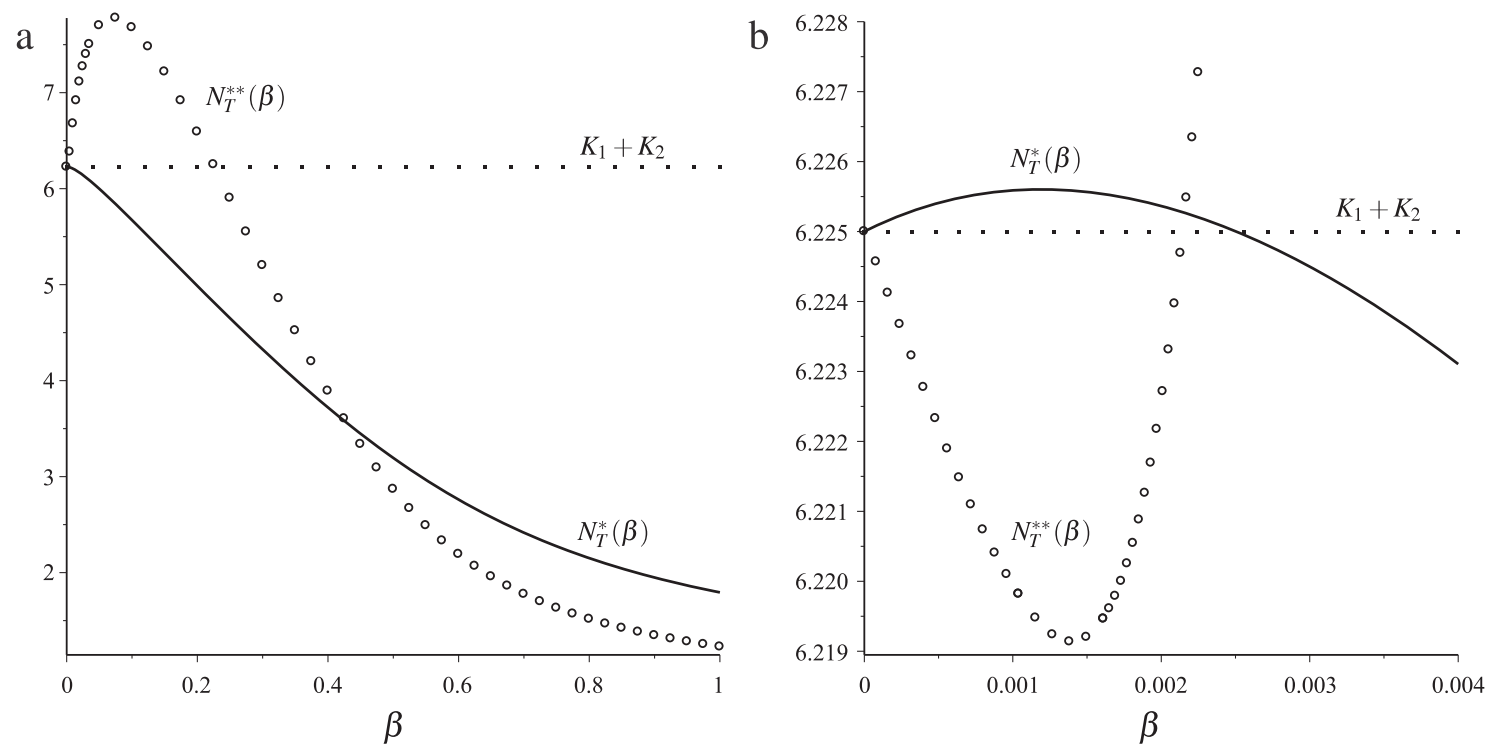

Fig. 4. Similar plots to Fig. 3 with different parameter values (see Tables 2 and 3). Panel (b) is a zoom of (a) in a narrow range of very small values of $\beta$. 

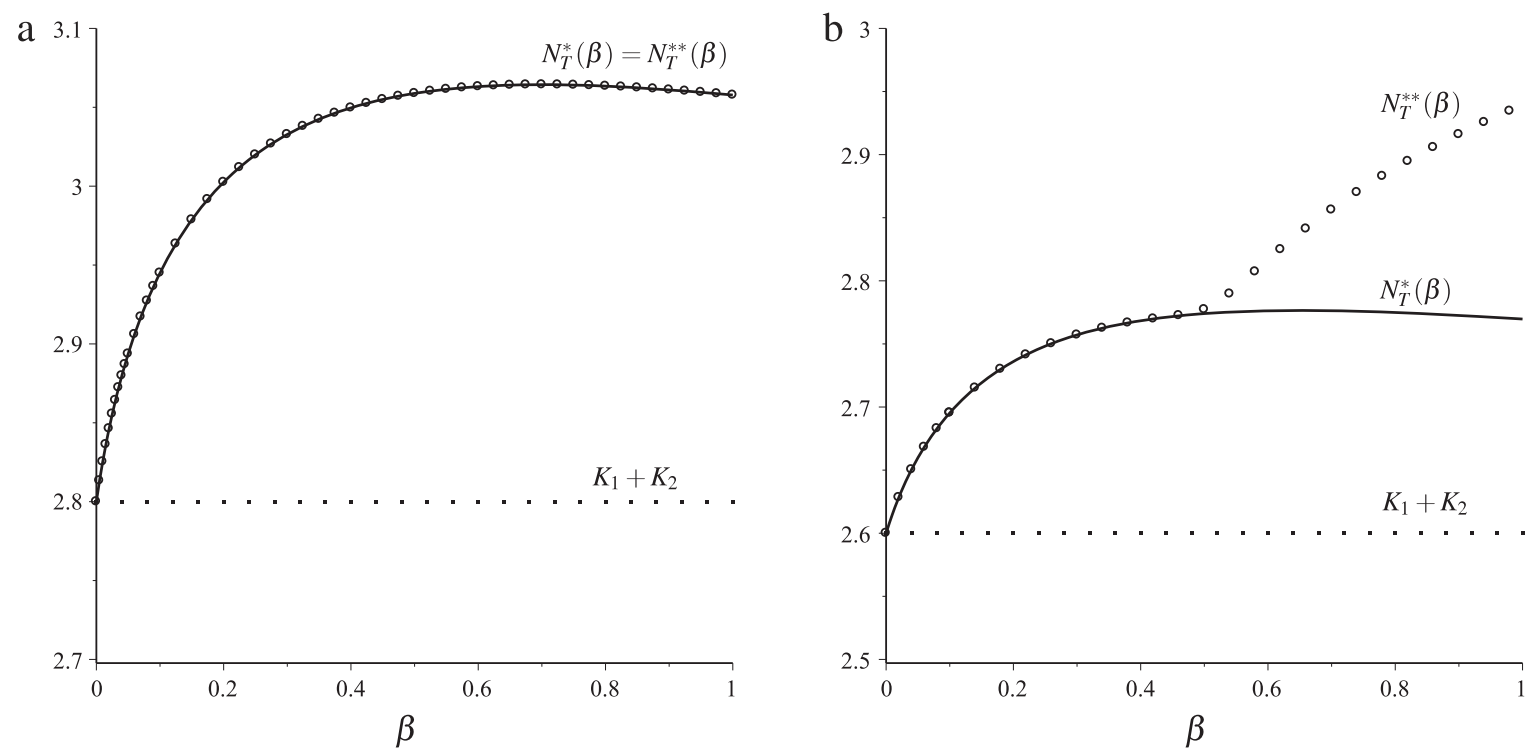

Fig. 5. Total consumer population as a function of migration when the consumer only can disperse. $N_{T}^{*}$ (solid curve) for the coupled-logistic reduced model, $N_{T}^{* *}$ (curve with circles) for the complete mechanistic model. See Table 4 for the parameter values and text in Section 4.2 for explanations.
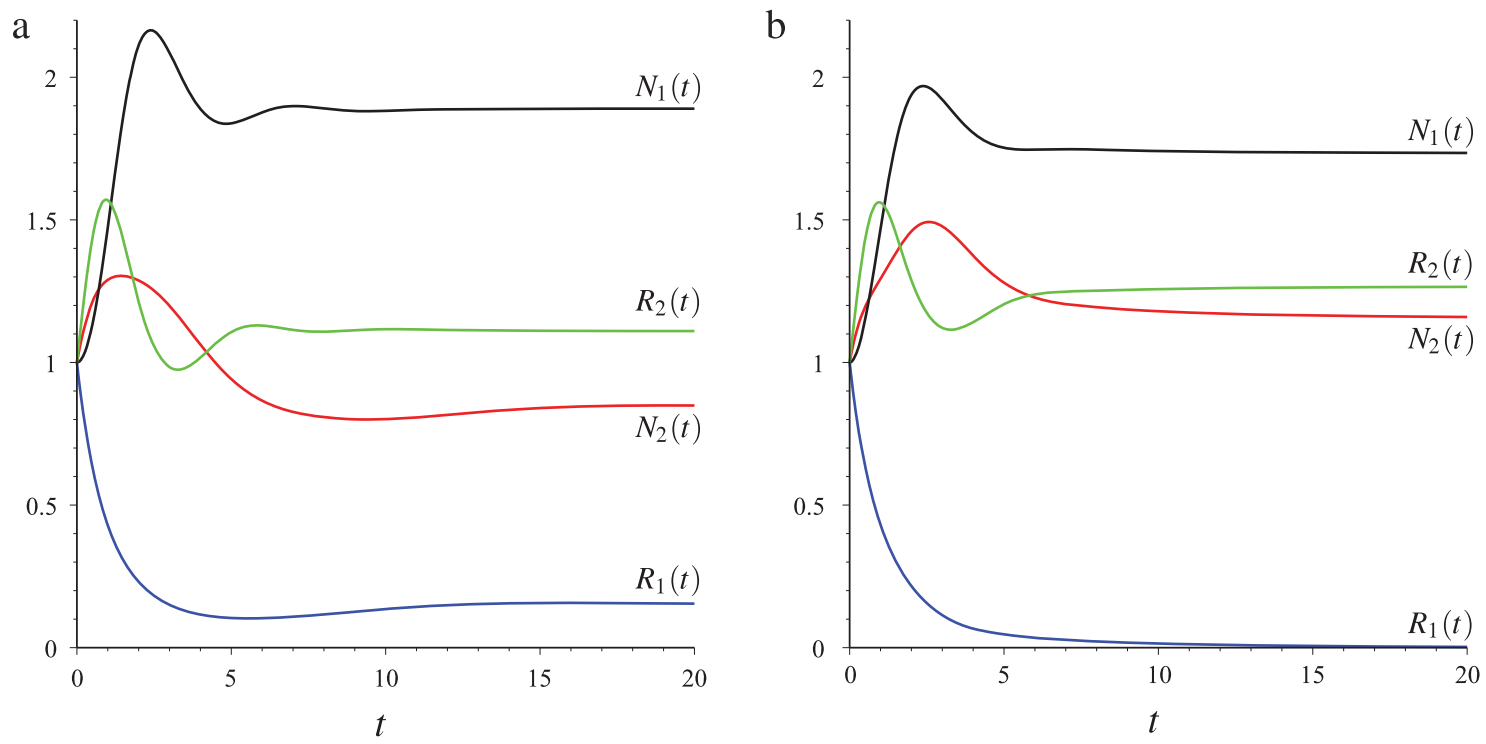

Fig. 6. Population abundances of $R_{1}(t), R_{2}(t), N_{1}(t)$, and $N_{2}(t)$ for the parameter values of Fig. $5(\mathrm{~b})$, with initial conditions $R_{1}(0)=R_{2}(0)=N_{1}(0)=N_{2}(0)=1$. (a) $\beta=0.2$, all species are present at equilibrium. (b) $\beta=0.8, R_{1}$ becomes extinct.

2. The simplest function of $N$ that decreases to 0 is $\mu(N)=$ $r\left(1-\frac{N}{K}\right)$.

3. Eq. (1) is a good approximation of some more complicated model.

4. The fit of the logistic model (1) to actual population dynamics is often fairly good.

From the above considerations, it seems natural to model migrations between two patches directly as in system (2). However, the correct generalization depends on the mechanisms that underly the logistic growth and that are not specified in the items 1 to 4 above. In the examples we have studied, we have shown that the patch version (2) could be incorrect.

Nevertheless, it can be correct under specific assumptions. For instance, the logistic model can be derived from the following mechanism, different from those of Sections 3 and 4. Assume that the population basically follows exponential growth:

$\frac{d N}{d t}=r N$ and that some proportion of the encounters between two individuals lead to mortality. In this case, if we also assume perfect mixing, the number of individuals dying during a small time interval $d t$ is simply proportional to the product $N^{2} d t$. Subtracting this mortality, we obtain the equation

$\frac{d N}{d t}=r N-\lambda N^{2}=r N\left(1-\frac{\lambda}{r} N\right)$,

which is a logistic (with carrying capacity $K=r / \lambda$ ). This is a mechanistic derivation of the logistic equation that assumes direct intraspecific interference.

Now, if we consider two patches with linear dispersal between them, we can assume the same mechanism and build the twopatch model (2) directly as a whole. In this case, we can be confident about the predictions of model (2), which is the traditional two-patch generalization of the logistic model.

If we accept the two-patch logistic model, our complete mathematical analysis summarized by Figs. 1 and 2 has determined the 
exact conditions under which fragmentation (associated to dispersal) increases the total equilibrium population size. This occurs in $\mathscr{J}_{0}$ for all migration rates (Fig. 2(a)) and in $\mathscr{J}_{1}$ for the lower migration rates (Fig. 2(c)). Thus, a necessary condition is $r_{2}>r_{1}$ (which is not always sufficient). Recalling that $K_{2}>K_{1}$, this means that, when the "good" patch 2 is the better one both in terms of carrying capacity and in terms of intrinsic growth rate, fragmentation can indeed have a beneficial effect. Fragmentation is always detrimental if carrying capacity and growth rate are negatively correlated, i.e., if $K_{2}>K_{1}$ and $r_{2}<r_{1}$. One may reasonably assume that this condition occurs in nature much less frequently than positive correlation (i.e., $K_{2}>K_{1}$ and $r_{2}>r_{1}$ ). Therefore, our analysis confirms the earlier partial results of other authors (e.g., Freedman and Waltman, 1977; Holt, 1985; DeAngelis and Zhang, 2014) who suggested that, in general, fragmentation was beneficial.

In particular, this will always be the case with the parameterization (19) of the logistic equation. In this case, $K$ is proportional to $r$ because $K=r / \lambda$. Therefore, if the fundamental cause of density dependence is the intrinsic interference $\lambda$, assumed to be the same in both patches, the fragmented logistic model (2) analysed in Section 2 is always on the border line between $\mathscr{J}_{0}$ and $\mathscr{J}_{1}$ of Fig. 1 . Fragmentation is always beneficial.

While this first message of our paper generally confirms previous results, our second message is more critical. We have shown that, if the logistic model is viewed as a mechanistic model (e.g., the two different mechanisms presented in Sections 3 and 4), then the correct two-patch generalization is different from the traditional reduced model (2). Moreover, the effect of fragmentation can be quite different from that predicted by the latter model. Figs. 3, 4, and 5 have shown that this effect can be either detrimental or beneficial, sometimes in a direction opposite to that predicted by the traditional model (2).

This second message of our paper brings some new light to earlier criticisms of the logistic equation, especially in the parameterization of Eq. (1) (e.g., Kuno, 1991, Ginzburg, 1992). Particularly, the expression "carrying capacity" for $K$ is very unfortunate because it conveys the idea that it is an intrinsic environmental property. With this view, our results would lead to say that the total carrying capacity of a patchy environment is different from the sum of the patches' carrying capacities. Instead, $K$ must be better viewed as the asymptotic, maximal value of the population abundance. This question was notably discussed by Gabriel et al. (2005) and by Mallet (2012), who pointed out that it makes much more sense to write the logistic equation as in Eq. (19) because it makes clear that the asymptotic limit of population abundance is due to intraspecific competition. Moreover, historically, this was the original way in which Pierre-François Verhulst first wrote the logistic equation (Verhulst, 1838).

A last point to discuss is to ask how our results generalize to situations with more than two patches. We have found that the complete analysis of the simplest two-patch case shows that the outcome is by no way intuitive. The mathematical extension to $n$ patches $(n>2)$ is probably very intricate and is a challenge for further work. Still, we can be pretty much confident that our two main findings remain qualitatively valid: (1) under some conditions, but not always, the total equilibrium population can be higher than the sum of the local carrying capacities; $(2)$ the coupling of $n$ patches with local logistic dynamics gives different theoretical results from those of the detailed direct coupling of the underlying mechanisms.

\section{Acknowledgements}

Our ideas germinated during a stimulating workshop organized in August 2014 at the Centre Interfacultaire Bernoulli (CIB) of the Ecole Polytechnique Fédérale de Lausanne (EPFL) in Switzerland.
We thank Don DeAngelis for organizing the scientific programme of this workshop and for his constructive remarks on an earlier version of this article. We also thank two anonymous reviewers for their very careful reading of our first submitted version.

\section{Appendix A. Appendix to Section 2}

\section{A.1. Some formulas}

Let us first prove the following preliminary result.

Proposition 1. Let $\left(N_{1}^{*}(\beta), N_{2}^{*}(\beta)\right)$ be an equilibrium of (2).

1. If $K_{1}<K_{2}$ and $\beta>0$, then $K_{1}<N_{1}^{*}(\beta)<N_{2}^{*}(\beta)<K_{2}$ (i.e., (5) holds).

2. Let $N_{T}^{*}(\beta)=N_{1}^{*}(\beta)+N_{2}^{*}(\beta)$. Then

$$
\begin{aligned}
& N_{T}^{*}=K_{1}+K_{2}+\beta \frac{N_{2}^{*}-N_{1}^{*}}{\frac{r_{1}}{K_{1}} \frac{r_{2}}{K_{2}} N_{1}^{*} N_{2}^{*}}\left(\frac{r_{2}}{K_{2}} N_{2}^{*}-\frac{r_{1}}{K_{1}} N_{1}^{*}\right) \\
& \text { and } \\
& \begin{aligned}
\frac{d N_{T}^{*}}{d \beta}= & \frac{N_{2}^{*}-N_{1}^{*}}{B\left(N_{1}^{*}, N_{2}^{*}\right)} \\
& \times\left[\beta\left(\frac{N_{1}^{*}}{N_{2}^{*}}-\frac{N_{2}^{*}}{N_{1}^{*}}\right)+\frac{r_{2}}{K_{2}} N_{2}^{*}-\frac{r_{1}}{K_{1}} N_{1}^{*}\right]
\end{aligned}
\end{aligned}
$$

where $B\left(N_{1}, N_{2}\right)=\frac{r_{1}}{K_{1}} \frac{r_{2}}{K_{2}} N_{1} N_{2}+\beta\left[\frac{r_{1}}{K_{1}} \frac{N_{1}^{2}}{N_{2}}+\frac{r_{2}}{K_{2}} \frac{N_{2}^{2}}{N_{1}}\right]$.

Proof. Let us prove item 1, that is to say, that (5) holds. The equilibria are the solutions of the set of equations

$$
\left\{\begin{array}{l}
0=r_{1} N_{1}\left(1-\frac{N_{1}}{K_{1}}\right)+\beta\left(N_{2}-N_{1}\right), \\
0=r_{2} N_{2}\left(1-\frac{N_{2}}{K_{2}}\right)+\beta\left(N_{1}-N_{2}\right) .
\end{array}\right.
$$

Solving the first equation for $N_{2}$ and the second for $N_{1}$ yields that the equilibria are the nonnegative intersections of the two parabolas $\mathscr{P}_{1}$ and $\mathscr{P}_{2}$ of equations $N_{2}=P_{1}\left(N_{1}\right)$ and $N_{1}=P_{2}\left(N_{2}\right)$, where the functions $P_{1}$ and $P_{2}$ are defined by

$$
\begin{aligned}
& P_{1}\left(N_{1}\right)=N_{1}-\frac{r_{1}}{\beta} N_{1}\left(1-\frac{N_{1}}{K_{1}}\right), \\
& P_{2}\left(N_{2}\right)=N_{2}-\frac{r_{2}}{\beta} N_{2}\left(1-\frac{N_{2}}{K_{2}}\right) .
\end{aligned}
$$

These parabolas are simply the isoclines $\dot{N}_{1}=0$ and $\dot{N}_{2}=0$. The isoclines intersect at $(0,0)$ and at $E=\left(N_{1}^{*}, N_{2}^{*}\right)$. Since $P_{1}\left(K_{1}\right)=K_{1}$, the point $A=\left(K_{1}, K_{1}\right)$ belongs to $\mathscr{P}_{1}$. Since $P_{2}\left(K_{2}\right)=K_{2}$, the point $B=\left(K_{2}, K_{2}\right)$ belongs to $\mathscr{P}_{2}$. Hence, the equilibrium $E$ belongs to the triangle $A B C$, where $C=\left(K_{1}, K_{2}\right)$ (see Fig. A.7). Thus $K_{1}<N_{1}^{*}<$ $N_{2}^{*}<K_{2}$, which is (5).

Let us now prove item 2. The proof of (A.1) is as follows. At the equilibrium $\left(N_{1}^{*}, N_{2}^{*}\right)$, one has:

$$
\left\{\begin{array}{l}
0=\frac{r_{1}}{K_{1}} N_{1}^{*}\left(K_{1}-N_{1}^{*}\right)+\beta\left(N_{2}^{*}-N_{1}^{*}\right), \\
0=\frac{r_{2}}{K_{2}} N_{2}^{*}\left(K_{2}-N_{2}^{*}\right)+\beta\left(N_{1}^{*}-N_{2}^{*}\right) .
\end{array}\right.
$$

Dividing the first equation by $\frac{r_{1}}{K_{1}} N_{1}^{*}$, the second by $\frac{r_{2}}{K_{2}} N_{2}^{*}$, and adding the two, one gets:

$K_{1}+K_{2}-\left(N_{1}^{*}+N_{2}^{*}\right)+\beta \frac{N_{2}^{*}-N_{1}^{*}}{\frac{r_{1}}{K_{1}} N_{1}^{*}}+\beta \frac{N_{1}^{*}-N_{2}^{*}}{\frac{r_{2}}{K_{2}} N_{2}^{*}}=0$. 

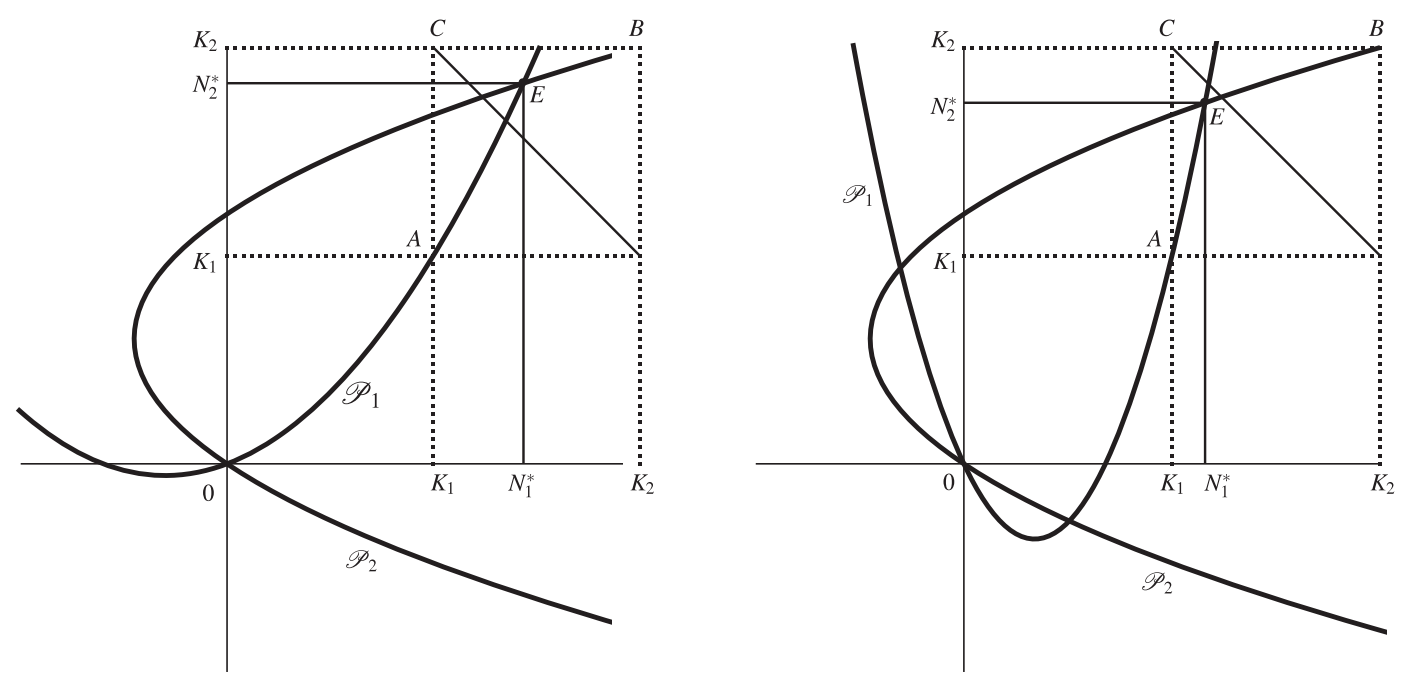

Fig. A.7. Phase-plane diagram for equation system (2). $E=\left(N_{1}^{*}, N_{2}^{*}\right)$, the positive intersection of the nullclines $\mathscr{P}_{1}$ and $\mathscr{P} 2$, is a stable equilibrium and 0 is an unstable one. Parameter values: $K_{1}=1, K_{2}=2, r_{2}=2, \beta=0.8$. Left: $r_{1}=0.5$, corresponding to the case $N_{T}^{*}>K_{1}+K_{2}$. Right: $r_{1}=2.5$, corresponding to the case $N_{T}^{*}<K_{1}+K_{2}$.

Hence

$N_{1}^{*}+N_{2}^{*}=K_{1}+K_{2}+\beta \frac{N_{2}^{*}-N_{1}^{*}}{\frac{r_{1}}{K_{1}} \frac{r_{2}}{K_{2}} N_{1}^{*} N_{2}^{*}}\left(\frac{r_{2}}{K_{2}} N_{2}^{*}-\frac{r_{1}}{K_{1}} N_{1}^{*}\right)$

which is (A.1).

The proof of (A.2) uses the implicit function theorem. Since $N_{1}^{*}(\beta)$ and $N_{2}^{*}(\beta)$ satisfy (A.3), one has

$f_{i}\left(N_{1}^{*}(\beta), N_{2}^{*}(\beta), \beta\right)=0, \quad i=1,2$,

where

$f_{i}\left(N_{1}, N_{2}, \beta\right)=r_{i} N_{i}\left(1-\frac{N_{i}}{K_{i}}\right)+\beta\left(N_{j}-N_{i}\right), \quad i, j=1,2, j \neq i$.

The total derivatives of (A.6) with respect to $\beta$ are

$$
\begin{aligned}
\frac{d f_{i}\left(N_{1}^{*}(\beta), N_{2}^{*}(\beta), \beta\right)}{d \beta}= & \frac{\partial f_{i}}{\partial N_{1}}\left(N_{1}^{*}(\beta), N_{2}^{*}(\beta), \beta\right) \frac{d N_{1}^{*}(\beta)}{d \beta} \\
& +\frac{\partial f_{i}}{\partial N_{2}}\left(N_{1}^{*}(\beta), N_{2}^{*}(\beta), \beta\right) \frac{d N_{2}^{*}(\beta)}{d \beta} \\
& +\frac{\partial f_{i}}{\partial \beta}\left(N_{1}^{*}(\beta), N_{2}^{*}(\beta), \beta\right)=0
\end{aligned}
$$

This is a linear system in $\frac{d N_{1}^{*}(\beta)}{d \beta}$ and $\frac{d N_{2}^{*}(\beta)}{d \beta}$ that can be solved to give

$\left[\begin{array}{l}\frac{d N_{1}^{*}}{d \beta}(\beta) \\ \frac{d N_{2}^{*}}{d \beta}(\beta)\end{array}\right]=-A^{-1}\left[\begin{array}{l}N_{2}^{*}(\beta)-N_{1}^{*}(\beta) \\ N_{1}^{*}(\beta)-N_{2}^{*}(\beta)\end{array}\right]$,

where

$A=\left[\begin{array}{cc}r_{1}\left(1-\frac{N_{1}^{*}(\beta)}{K_{1}}\right)-\beta-\frac{r_{1}}{K_{1}} N_{1}^{*}(\beta) & \beta \\ \beta & r_{2}\left(1-\frac{N_{2}^{*}(\beta)}{K_{2}}\right)-\beta-\frac{r_{2}}{K_{2}} N_{2}^{*}(\beta)\end{array}\right]$.

Using (A.5), we have

$r_{1}\left(1-\frac{N_{1}^{*}}{K_{1}}\right)-\beta=-\beta \frac{N_{2}^{*}}{N_{1}^{*}}, \quad r_{2}\left(1-\frac{N_{2}^{*}}{K_{2}}\right)-\beta=-\beta \frac{N_{1}^{*}}{N_{2}^{*}}$.
Using these formulas, and after some algebraic manipulation, Eq. (A.7) reduces to

$$
\begin{aligned}
{\left[\begin{array}{c}
\frac{d N_{1}^{*}}{d \beta} \\
\frac{d N_{2}^{*}}{d \beta}
\end{array}\right]=} & \frac{1}{B\left(N_{1}^{*}, N_{2}^{*}\right)}\left[\begin{array}{cc}
\beta \frac{N_{1}^{*}}{N_{2}^{*}}+\frac{r_{2}}{K_{2}} N_{2}^{*} & \beta \\
\beta & \beta \frac{N_{2}^{*}}{N_{1}^{*}}+\frac{r_{1}}{K_{1}} N_{1}^{*}
\end{array}\right] \\
& \times\left[\begin{array}{l}
N_{2}^{*}-N_{1}^{*} \\
N_{1}^{*}-N_{2}^{*}
\end{array}\right] .
\end{aligned}
$$

Therefore

$$
\begin{aligned}
\frac{d N_{1}^{*}}{d \beta}= & \frac{1}{B\left(N_{1}^{*}, N_{2}^{*}\right)} \\
& \times\left[\left(\beta \frac{N_{1}^{*}}{N_{2}^{*}}+\frac{r_{2}}{K_{2}} N_{2}^{*}\right)\left(N_{2}^{*}-N_{1}^{*}\right)+\beta\left(N_{1}^{*}-N_{2}^{*}\right)\right], \\
\frac{d N_{2}^{*}}{d \beta}= & \frac{1}{B\left(N_{1}^{*}, N_{2}^{*}\right)} \\
& \times\left[\left(\beta \frac{N_{2}^{*}}{N_{1}^{*}}+\frac{r_{1}}{K_{1}} N_{1}^{*}\right)\left(N_{1}^{*}-N_{2}^{*}\right)+\beta\left(N_{2}^{*}-N_{1}^{*}\right)\right] .
\end{aligned}
$$

Adding the two equations, one obtains (A.2).

Remark. The stability study of $E=\left(N_{1}^{*}, N_{2}^{*}\right)$ comes from the analysis of the variational matrix. See Freedman and Waltman (1977) and DeAngelis et al. (1979), where it is proved that $E$ is stable. Actually, $E$ is globally asymptotically stable (Holt, 1985). Graphically, the two parabolic isoclines in Fig. A.7 are attractive $\left(\mathscr{P}_{1}\right.$ horizontally and $\mathscr{P}_{2}$ vertically). $\mathscr{P}_{1}$ can only be crossed vertically and $\mathscr{P}_{2}$ horizontally. It is therefore easy to follow the general direction of trajectories in the positive quadrant of this figure and to understand that they all lead to the equilibrium point $E$.

\section{A.2. Comparison of $N_{T}^{*}$ and $K_{1}+K_{2}$}

In this section, we explain why, in general, the total equilibrium population in the system of coupled logistic growths (2) is different from the sum of the carrying capacities. More precisely, we give the exact conditions under which $N_{T}^{*}>K_{1}+K_{2}$. Recall that, if $K_{1}=K_{2}$, then $N_{1}^{*}=K_{1}$ and $N_{2}^{*}=K_{2}$ for any $\beta \geq 0$. Therefore $N_{T}^{*}=K_{1}+K_{2}$ for any $\beta \geq 0$. When $K_{1}$ is not equal to $K_{2}$, we have the following result, where $N_{T}^{*}(\beta)$ is studied as a function of the migration rate $\beta$. 
Proposition 2. We recall the assumption that $K_{1}<K_{2}$.

1. If $\frac{r_{2}}{K_{2}} \geq \frac{r_{1}}{K_{1}}$, then $N_{T}^{*}(\beta)>K_{1}+K_{2}$ for any $\beta>0$.

2. If $\frac{r_{2}}{K_{2}}<\frac{r_{1}}{K_{1}}$ and $r_{2}>r_{1}$, then $N_{T}^{*}(\beta)>K_{1}+K_{2}$ for $0<\beta<\beta_{0}$ and

$N_{T}^{*}(\beta)<K_{1}+K_{2}$ for $\beta>\beta_{0}$, where $\beta_{0}>0$ is defined by

$\beta_{0}=\frac{r_{2}-r_{1}}{\frac{K_{2}}{r_{2}}-\frac{K_{1}}{r_{1}}} \frac{1}{\frac{r_{2}}{K_{2}}+\frac{r_{1}}{K_{1}}}$.

3. If $r_{2} \leq r_{1}$, then $N_{T}^{*}(\beta)<K_{1}+K_{2}$ for any $\beta>0$.

Proof. From (A.1), we can study the cases 1 and 3 of Proposition 2.

- If $\frac{r_{2}}{K_{2}} \geq \frac{r_{1}}{K_{1}}$, then, using (5), we have

$\frac{r_{2}}{K_{2}} N_{2}^{*}-\frac{r_{1}}{K_{1}} N_{1}^{*} \geq \frac{r_{1}}{K_{1}} N_{2}^{*}-\frac{r_{1}}{K_{1}} N_{1}^{*}=\frac{r_{1}}{K_{1}}\left(N_{2}^{*}-N_{1}^{*}\right)$.

Therefore, using $N_{2}^{*}>N_{1}^{*}$ and (A.1), we have $N_{T}^{*}>K_{1}+K_{2}$.

- If $r_{2} \leq r_{1}$, then, using (5), we have $\frac{N_{2}^{*}}{K_{2}}<1$ and $\frac{N_{1}^{*}}{K_{1}}>1$, so that $\frac{r_{2}}{K_{2}} N_{2}^{*}-\frac{r_{1}}{K_{1}} N_{1}^{*}=r_{2} \frac{N_{2}^{*}}{K_{2}}-r_{1} \frac{N_{1}^{*}}{K_{1}}<r_{2}-r_{1} \leq 0$.

Therefore, using $N_{2}^{*}>N_{1}^{*}$ and (A.1), we have $N_{T}^{*}<K_{1}+K_{2}$.

The study of the case 2 of Proposition 2 requires both (A.1) and (A.2). From (A.1) we deduce that $N_{T}^{*}(\beta)=K_{1}+K_{2}$ for $\beta>0$, if and only if

$\frac{r_{2}}{K_{2}} N_{2}^{*}-\frac{r_{1}}{K_{1}} N_{1}^{*}=0$.

Using (A.2), we see that (A.8) necessarily implies that $\frac{d N_{T}^{*}}{d \beta}(\beta)<$ 0 . Hence, we can have $N_{T}^{*}(\beta)=K_{1}+K_{2}$ for at most one value of $\beta>0$. For such value of $\beta,\left(N_{1}^{*}, N_{2}^{*}\right)$ is a solution of the set of linear equations formed by (A.8) and the condition

$N_{1}^{*}+N_{2}^{*}=K_{1}+K_{2}$.

Solving (A.8)-(A.9), we obtain

$N_{1}^{*}=\frac{K_{1}}{r_{1}} \frac{K_{1}+K_{2}}{\frac{K_{1}}{r_{1}}+\frac{K_{2}}{r_{2}}}, \quad N_{2}^{*}=\frac{K_{2}}{r_{2}} \frac{K_{1}+K_{2}}{\frac{K_{1}}{r_{1}}+\frac{K_{2}}{r_{2}}}$.

Using (A.5), we obtain that

$\beta=\frac{r_{2}-r_{1}}{\frac{K_{2}}{r_{2}}-\frac{K_{1}}{r_{1}}} \frac{1}{\frac{r_{2}}{K_{2}}+\frac{r_{1}}{K_{1}}}$.

We conclude that $N_{T}^{*}(\beta)=K_{1}+K_{2}$ if and only if $\beta$ is equal to this value, and that $N_{T}^{*}(\beta)<K_{1}+K_{2}$ if and only if $\beta$ is greater than this value.

\section{A.3. Perfect mixing}

The behaviour of the system for perfect mixing $(\beta \rightarrow \infty)$ is given by the following result.

Proposition 3. Let $\left(N_{1}(t, \beta), N_{2}(t, \beta)\right)$ be a solution of (2) with initial condition $\left(N_{10}, N_{20}\right)$. When $\beta \rightarrow \infty$, then, with the exception of a small initial interval, $N_{1}(t, \beta)$ and $N_{2}(t, \beta)$ are both approximated by the solution $N(t)$ of the logistic equation (1), where $r=\frac{r_{1}+r_{2}}{2}$ and $K=\frac{r_{1}+r_{2}}{r_{1} / K_{1}+r_{2} / K_{2}}$, and with initial condition $N_{0}=\frac{N_{10}+N_{20}}{2}$.
Proof. Let $N=\frac{N_{1}+N_{2}}{2}$. We can rewrite (2) using the variables $N_{1}$ and $N$ (notice that $\left.\stackrel{2}{N}_{2}=2 N-N_{1}\right)$ :

$$
\left\{\begin{array}{l}
\frac{d N_{1}}{d t}=r_{1} N_{1}\left(1-\frac{N_{1}}{K_{1}}\right)+2 \beta\left(N-N_{1}\right), \\
\frac{d N}{d t}=\frac{1}{2}\left[r_{1} N_{1}\left(1-\frac{N_{1}}{K_{1}}\right)+r_{2}\left(2 N-N_{1}\right)\left(1-\frac{2 N-N_{1}}{K_{2}}\right)\right] .
\end{array}\right.
$$

The system (A.10) is a slow and fast system whose slow variable is $N$ and fast variable is $N_{1}$. We use the Tikhonov theorem (Tikhonov, 1952; Wasow, 1976; Lobry et al., 1998) to show that, in the limit $\beta \rightarrow \infty$, the solutions of (A.10) are approximated by the solutions of the reduced model. The reduced model is obtained as follows.

We first consider the dynamics of the fast variable $N_{1}$ in the time scale $\tau=\beta t$, which is

$$
\frac{d N_{1}}{d \tau}=\frac{1}{\beta} r_{1} N_{1}\left(1-\frac{N_{1}}{K_{1}}\right)+2\left(N-N_{1}\right) .
$$

The fast equation

$\frac{d N_{1}}{d \tau}=2\left(N-N_{1}\right)$

is obtained from the previous one by setting $1 / \beta=0$ in the right hand side.

Since $N_{1}=N$ is an asymptotically stable equilibrium of the fast equation, the Tikhonov theorem applies and tells us that the reduced model is obtained by replacing, in the second equation of (A.10), the fast variable $N_{1}$ by the equilibrium $N_{1}=N$ of the fast equation. One obtains

$\frac{d N}{d t}=\frac{r_{1}+r_{2}}{2} N\left(1-N \frac{r_{1} / K_{1}+r_{2} / K_{2}}{r_{1}+r_{2}}\right)$

which is the logistic equation with parameters $r$ and $K$ as given by the formulas in the present Proposition.

This reduction method is also known as the quasi-steady state approximation, since $N_{1}$ is replaced by the quasi-steady state $N_{1}=$ $N$ of the fast equation (A.11).

Eq. (A.12) is simply a logistic equation whose positive equilibrium is given by

$N^{*}=\frac{r_{1}+r_{2}}{r_{1} / K_{1}+r_{2} / K_{2}}$.

Hence, in the limit $\beta \rightarrow \infty$, we get $N_{1}^{*}(+\infty)=N_{2}^{*}(+\infty)=N^{*}$, so that $N_{T}^{*}(+\infty)=N_{1}^{*}(+\infty)+N_{2}^{*}(+\infty)$ is given by

$N_{T}^{*}(+\infty)=2 \frac{r_{1}+r_{2}}{r_{1} / K_{1}+r_{2} / K_{2}}$,

which is the same result as (3).

Remark. The property $N_{1}^{*}(+\infty)=N_{2}^{*}(+\infty)=\frac{r_{1}+r_{2}}{r_{1} / K_{1}+r_{2} / K_{2}}$ had already been obtained by Freedman and Waltman (1977, their Theorem 3.1) by a direct computation on the Eqs. (A.3). See also Holt (1985, his Section 2.3). We have obtained here this formula from the model (A.12) to which the model (2) reduces in the limit $\beta \rightarrow \infty$. This approach is more general than the direct computations used by Freedman and Waltman (1977) and will be useful for other models considered in this paper.

A more complete understanding of the effect of migration is provided by the following proposition, which gives additional information on the derivative of $N_{T}^{*}(\beta)$ with respect to $\beta$. 
Proposition 4. 1. $\frac{d N_{T}^{*}}{d \beta}(0)=\left(K_{1}-K_{2}\right)\left(\frac{1}{r_{2}}-\frac{1}{r_{1}}\right)=\left(K_{1}-K_{2}\right) \frac{r_{1}-r_{2}}{r_{1} r_{2}}$.

2. If $N_{T}^{*}(\beta)<K_{1}+K_{2}$, then $\frac{d N_{T}^{*}}{d \beta}(\beta)<0$.

Proof. Using $N_{1}^{*}(0)=K_{1}, N_{2}^{*}(0)=K_{2}$ in (A.2), we get

$\frac{d N_{T}^{*}}{d \beta}(0)=\left(\frac{1}{r_{2}}-\frac{1}{r_{1}}\right)\left(K_{1}-K_{2}\right)$.

This is item 1 of Proposition 4.

From (A.1) we deduce that $N_{T}^{*}(\beta)<K_{1}+K_{2}$ if and only if

$\frac{r_{2}}{K_{2}} N_{2}^{*}-\frac{r_{1}}{K_{1}} N_{1}^{*}<0$.

Using (A.2), we see that this condition necessarily implies that $\frac{d N_{T}^{*}}{d \beta}(\beta)<0$. This is item 2 of Proposition 4 .

Using (A.13) and Proposition 4, we can notice that

- $\mathscr{J}_{0}$ is characterized by the condition $N_{T}^{*}(+\infty) \geq K_{1}+K_{2}$,

- $\mathscr{J}_{1}$ is characterized by the conditions $N_{T}^{*}(+\infty)<K_{1}+K_{2}$ and $\frac{d N_{T}^{*}}{d \beta}(0)>0$,

- $\mathscr{J}_{2}$ is characterized by the condition $\frac{d N_{T}^{*}}{d \beta}(0) \leq 0$.

\section{Appendix B. Appendix to Section 4.1}

We assume in this section that the dispersion rates of the consumer and the resource are equal. The mathematical model is system (17) with $\alpha=\beta$, that is:

$$
\left\{\begin{array}{l}
\frac{d R_{1}}{d t}=\left[s_{1}\left(1-\frac{R_{1}}{L_{1}}\right)-a_{1} N_{1}\right] R_{1}+\beta\left(R_{2}-R_{1}\right), \\
\frac{d R_{2}}{d t}=\left[s_{2}\left(1-\frac{R_{2}}{L_{2}}\right)-a_{2} N_{2}\right] R_{2}+\beta\left(R_{1}-R_{2}\right), \\
\frac{d N_{1}}{d t}=\varepsilon_{1}\left(b_{1} R_{1}-q_{1}\right) N_{1}+\beta\left(N_{2}-N_{1}\right), \\
\frac{d N_{2}}{d t}=\varepsilon_{2}\left(b_{2} R_{2}-q_{2}\right) N_{2}+\beta\left(N_{1}-N_{2}\right) .
\end{array}\right.
$$

This system can have many equilibria, whose analytical study is difficult (if not impossible) and is beyond the scope of this paper. As already mentioned in Section 4, computer simulations show that (B.1) has a globally stable equilibrium. Assuming that this equilibrium exists for each value of $\beta$ and is positive, we can consider, as in the previous section, its dependence with respect to $\beta$. Let $\left(R_{1}^{* *}(\beta), R_{2}^{* *}(\beta), N_{1}^{* *}(\beta), N_{2}^{* *}(\beta)\right)$ be the positive and stable equilibrium of (B.1). Let $\left(N_{1}^{*}(\beta), N_{2}^{*}(\beta)\right)$ be the globally stable equilibrium of the corresponding reduced model (14). We consider here some properties of $N_{T}^{*}(\beta)$ and $N_{T}^{* *}(\beta)$ as functions of the migration rate $\beta$.

From Proposition 4 and (A.13), we have

$$
\begin{aligned}
& \frac{d N_{T}^{*}}{d \beta}(0)=\left(K_{1}-K_{2}\right)\left(\frac{1}{r_{2}}-\frac{1}{r_{1}}\right), \\
& N_{T}^{*}(+\infty)=2 \frac{r_{1}+r_{2}}{r_{1} / K_{1}+r_{2} / K_{2}},
\end{aligned}
$$

where $r_{i}$ and $K_{i}$ are given by Eqs. (15)-(16).

\section{B.1. Perfect mixing}

The behaviour of (B.1) for perfect mixing $(\beta \rightarrow \infty)$ is given by the following result.
Proposition 5. Let $\left(R_{1}(t, \beta), R_{2}(t, \beta), N_{1}(t, \beta), N_{2}(t, \beta)\right)$ be a solution of (B.1) with initial condition $\left(R_{10}, R_{20}, N_{10}, N_{20}\right)$. When $\beta \rightarrow$ $\infty$, then, with the exception of a small initial interval, $R_{1}(t, \beta)$ and $R_{2}(t, \beta)$ are both approximated by $R(t)$, and $N_{1}(t, \beta)$ and $N_{2}(t, \beta)$ are both approximated by $N(t)$, where $(R(t), N(t))$ is the solution of the MacArthur single-patch model (12) where

$$
\begin{aligned}
& s=\frac{s_{1}+s_{2}}{2}, \quad L=\frac{s_{1}+s_{2}}{s_{1} / L_{1}+s_{2} / L_{2}}, \quad a=\frac{a_{1}+a_{2}}{2}, \\
& \varepsilon=\frac{\varepsilon_{1}+\varepsilon_{2}}{2}, \quad w=\frac{2}{a_{1}+a_{2}} \frac{\varepsilon_{1} b_{1}+\varepsilon_{2} b_{2}}{\varepsilon_{1}+\varepsilon_{2}}, \\
& q=\frac{\varepsilon_{1} q_{1}+\varepsilon_{2} q_{2}}{\varepsilon_{1}+\varepsilon_{2}},
\end{aligned}
$$

and with initial condition $R_{0}=\frac{R_{10}+R_{20}}{2}$ and $N_{0}=\frac{N_{10}+N_{20}}{2}$.

Proof. We use here the singular perturbation analysis outlined in Appendix A.3 to obtain the behaviour of the system as $\beta \rightarrow \infty$. Let

$R=\frac{R_{1}+R_{2}}{2}, \quad N=\frac{N_{1}+N_{2}}{2}$.

We can rewrite (17) using the variables $R_{1}, N_{1}$ and $R, N$ (using $R_{2}=2 R-R_{1}$ and $N_{2}=2 N-N_{1}$ ):

$$
\left\{\begin{aligned}
\frac{d R_{1}}{d t} & =\left[s_{1}\left(1-\frac{R_{1}}{L_{1}}\right)-a_{1} N_{1}\right] R_{1}+2 \beta\left(R-R_{1}\right) \\
\frac{d N_{1}}{d t} & =\varepsilon_{1}\left(b_{1} R_{1}-q_{1}\right) N_{1}+2 \beta\left(N-N_{1}\right) \\
\frac{d R}{d t} & =\frac{1}{2}\left[s_{1}\left(1-\frac{R_{1}}{L_{1}}\right)-a_{1} N_{1}\right] R_{1} \\
& +\frac{1}{2}\left[s_{2}\left(1-\frac{2 R-R_{1}}{L_{2}}\right)-a_{2}\left(2 N-N_{1}\right)\right] \\
& \times\left(2 R-R_{1}\right) \\
\frac{d N}{d t} & =\frac{1}{2}\left[\varepsilon_{1}\left(b_{1} R_{1}-q_{1}\right) N_{1}+\varepsilon_{2}\left(b_{2}\left(2 R-R_{2}\right)\right.\right. \\
& \left.\left.-q_{2}\right)\left(2 N-N_{2}\right)\right] .
\end{aligned}\right.
$$

System (B.3) is a slow and fast system whose slow variables are $R$ and $N$ and fast variables are $R_{1}$ and $N_{1}$. In the limit $\beta \rightarrow \infty$, we can replace the fast variables $R_{1}$ and $N_{1}$ in the third and fourth equations by their quasi-steady state approximations $R_{1}=R$ and $N_{1}=N$ obtained from the first and second equations. We obtain

$\left\{\begin{array}{l}\frac{d R}{d t}=\left[\frac{s_{1}+s_{2}}{2}\left(1-R \frac{s_{1} / L_{1}+s_{2} / L_{2}}{s_{1}+s_{2}}\right)-\frac{a_{1}+a_{2}}{2} N\right] R, \\ \frac{d N}{d t}=\frac{1}{2}\left[\left(\varepsilon_{1} b_{1}+\varepsilon_{2} b_{2}\right) R-\left(\varepsilon_{1} q_{1}+\varepsilon_{2} q_{2}\right)\right] N .\end{array}\right.$

This is simply the MacArthur resource-consumer model (12) with parameters as given by the formulas in the present Proposition.

The positive equilibrium of (B.4) is given by

$$
R^{*}=\frac{\varepsilon_{1} q_{1}+\varepsilon_{2} q_{2}}{\varepsilon_{1} b_{1}+\varepsilon_{2} b_{2}}, \quad N^{*}=\frac{s_{1}+s_{2}}{a_{1}+a_{2}}\left(1-R^{*} \frac{\frac{s_{1}}{L_{1}}+\frac{s_{2}}{L_{2}}}{s_{1}+s_{2}}\right) .
$$

This equilibrium is positive if and only if $\frac{\varepsilon_{1} q_{1}+\varepsilon_{2} q_{2}}{\varepsilon_{1} b_{1}+\varepsilon_{2} b_{2}}<\frac{s_{1}+s_{2}}{\frac{s_{1}}{L_{1}}+\frac{s_{2}}{L_{2}}}$.

Hence, in the limit $\beta \rightarrow \infty$, we get $N_{1}^{* *}(+\infty)=N_{2}^{* *}(+\infty)=$ $N^{*}$, so that $N_{T}^{* *}(+\infty)=N_{1}^{* *}(+\infty)+N_{2}^{* *}(+\infty)$ is given by

$N_{T}^{* *}(+\infty)=2 \frac{s_{1}+s_{2}}{a_{1}+a_{2}}\left(1-\frac{\varepsilon_{1} q_{1}+\varepsilon_{2} q_{2}}{\varepsilon_{1} b_{1}+\varepsilon_{2} b_{2}} \frac{\frac{s_{1}}{L_{1}}+\frac{s_{2}}{L_{2}}}{s_{1}+s_{2}}\right)$. 


\section{B.2. Comparison of $N_{T}^{* *}$ and $N_{T}^{*}$ for small $\beta$}

A more complete understanding of the effect of migration is provided by the following proposition, which gives the derivative of $\frac{d N_{T}^{* *}}{d \beta}(0)$.

Proposition 6. We have

$\frac{d N_{T}^{* *}}{d \beta}(0)=\left(\frac{b_{2}}{a_{2} q_{2}}-\frac{b_{1}}{a_{1} q_{1}}\right)\left(\frac{q_{1}}{b_{1}}-\frac{q_{2}}{b_{2}}\right)+\frac{d N_{T}^{*}}{d \beta}(0)$.

Proof. Let $\left(R_{1}^{* *}(\beta), R_{2}^{* *}(\beta), N_{1}^{* *}(\beta), N_{2}^{* *}(\beta)\right)$ be a positive equilibrium of (B.1). Thus, it is a solution of the set of equations

$$
\left\{\begin{array}{l}
0=\left[s_{1}\left(1-\frac{R_{1}^{* *}}{L_{1}}\right)-a_{1} N_{1}^{* *}\right] R_{1}^{* *}+\beta\left(R_{2}^{* *}-R_{1}^{* *}\right) \\
0=\left[s_{2}\left(1-\frac{R_{2}^{* *}}{L_{2}}\right)-a_{2} N_{2}^{* *}\right] R_{2}^{* *}+\beta\left(R_{1}^{* *}-R_{2}^{* *}\right) \\
0=\varepsilon_{1}\left(b_{1} R_{1}^{* *}-q_{1}\right) N_{1}^{* *}+\beta\left(N_{2}^{* *}-N_{1}^{* *}\right) \\
0=\varepsilon_{2}\left(b_{2} R_{2}^{* *}-q_{2}\right) N_{2}^{* *}+\beta\left(N_{1}^{* *}-N_{2}^{* *}\right) .
\end{array}\right.
$$

As in Appendix A.1, we use the implicit function theorem and calculate the derivatives $\frac{d R_{i}^{* *}}{d \beta}(\beta)$ and $\frac{d N_{i}^{* *}}{d \beta}(\beta)$. We have

$$
\left[\begin{array}{l}
\frac{d R_{1}^{* *}}{d \beta}(\beta) \\
\frac{d R_{2}^{* *}}{d \beta}(\beta) \\
\frac{d N_{1}^{* *}}{d \beta}(\beta) \\
\frac{d_{2} N^{* *}}{d \beta}(\beta)
\end{array}\right]=-A(\beta)^{-1}\left[\begin{array}{c}
R_{2}^{* *}(\beta)-R_{1}^{* *}(\beta) \\
R_{1}^{* *}(\beta)-R_{2}^{* *}(\beta) \\
N_{2}^{* *}(\beta)-N_{1}^{* *}(\beta) \\
N_{1}^{* *}(\beta)-N_{2}^{* *}(\beta)
\end{array}\right],
$$

where

$A(\beta)=\left[\begin{array}{ll}A_{11}(\beta) & A_{12}(\beta) \\ A_{21}(\beta) & A_{22}(\beta)\end{array}\right]$,

and the matrices $A_{i j}(\beta)$ are given by

$A_{11}(\beta)=\left[\begin{array}{cc}B_{1}(\beta) & \beta \\ \beta & B_{2}(\beta)\end{array}\right]$,

$A_{12}(\beta)=\left[\begin{array}{cc}-a_{1} R_{1}^{* *}(\beta) & 0 \\ 0 & -a_{2} R_{2}^{* *}(\beta)\end{array}\right]$,

$A_{21}(\beta)=\left[\begin{array}{cc}\varepsilon_{1} b_{1} N_{1}^{* *}(\beta) & 0 \\ 0 & \varepsilon_{2} b_{2} N_{2}^{* *}(\beta)\end{array}\right]$

$A_{22}(\beta)=\left[\begin{array}{cc}\varepsilon_{1}\left(b_{1} R_{1}^{* *}(\beta)-q_{1}\right)-\beta & \beta \\ \beta & \varepsilon_{2}\left(b_{2} R_{2}^{* *}(\beta)-q_{2}\right)-\beta\end{array}\right]$,

with

$B_{i}(\beta)=-\frac{s_{i}}{L_{i}} R_{i}^{* *}(\beta)+s_{i}\left(1-\frac{R_{i}^{* *}(\beta)}{L_{i}}\right)-a_{i} N_{i}^{* *}(\beta)-\beta$.
Using $R_{i}^{* *}(0)=\frac{q_{i}}{b_{i}}$ and $N_{i}^{* *}(0)=\frac{s_{i}}{a_{i}} \frac{b_{i} L_{i}-q_{i}}{b_{i} L_{i}}=K_{i}$, and after some algebraic manipulation, for $\beta=0$, Eq. (B.8) reduces to

$\left[\begin{array}{l}\frac{d R_{1}^{* *}}{d \beta}(0) \\ \frac{d R_{2}^{* *}}{d \beta}(0) \\ \frac{d N_{1}^{* *}}{d \beta}(0) \\ \frac{d N_{2}^{* *}}{d \beta}(0)\end{array}\right]=\left[\begin{array}{cccc}0 & 0 & -\frac{1}{\varepsilon_{1} b_{1} K_{1}} & 0 \\ 0 & 0 & 0 & -\frac{1}{\varepsilon_{2} b_{2} K_{2}} \\ \frac{b_{1}}{a_{1} q_{1}} & 0 & \frac{1}{r_{1}} & 0 \\ 0 & \frac{b_{2}}{a_{2} q_{2}} & 0 & \frac{1}{r_{2}}\end{array}\right]\left[\begin{array}{c}\frac{q_{2}}{b_{2}}-\frac{q_{1}}{b_{1}} \\ \frac{q_{1}}{b_{1}}-\frac{q_{2}}{b_{2}} \\ K_{2}-K_{1} \\ K_{1}-K_{2}\end{array}\right]$,

where $r_{i}$ and $K_{i}$ are given by (15)-(16). Therefore

$\frac{d N_{1}^{* *}}{d \beta}(0)=\frac{b_{1}}{a_{1} q_{1}}\left(\frac{q_{2}}{b_{2}}-\frac{q_{1}}{b_{1}}\right)+\frac{1}{r_{1}}\left(K_{2}-K_{1}\right)$,

$\frac{d N_{2}^{* *}}{d \beta}(0)=\frac{b_{2}}{a_{2} q_{2}}\left(\frac{q_{1}}{b_{1}}-\frac{q_{2}}{b_{2}}\right)+\frac{1}{r_{2}}\left(K_{1}-K_{2}\right)$.

Adding these equations, one obtains

$\frac{d N_{T}^{* *}}{d \beta}(0)=\left(\frac{b_{2}}{a_{2} q_{2}}-\frac{b_{1}}{a_{1} q_{1}}\right)\left(\frac{q_{1}}{b_{1}}-\frac{q_{2}}{b_{2}}\right)+\left(K_{1}-K_{2}\right)\left(\frac{1}{r_{2}}-\frac{1}{r_{1}}\right)$.

Using (B.2), we obtain (B.6).

The formulas (B.2), (B.5), and (B.6) give the values of the derivatives at $\beta=0$ and the values at $\beta=\infty$ of the functions $N_{T}^{*}(\beta)$ and $N_{T}^{* *}(\beta)$. They show that $N_{T}^{*}(\beta)$ and $N_{T}^{* *}(\beta)$ are different from each other. The parameter values can be chosen in such way to display the typical behaviours of the examples considered in Section 4.2. See Figs. 3 and 4.

\section{Appendix C. Appendix to Section 4.2}

Here, we give the mathematical analysis of the complete resource-consumer model in the case in which the consumer alone can disperse. The mathematical model is system (17) with $\alpha$ set to 0 , that is:

$\left\{\begin{array}{l}\frac{d R_{1}}{d t}=\left[s_{1}\left(1-\frac{R_{1}}{L_{1}}\right)-a_{1} N_{1}\right] R_{1} \\ \frac{d R_{2}}{d t}=\left[s_{2}\left(1-\frac{R_{2}}{L_{2}}\right)-a_{2} N_{2}\right] R_{2} \\ \frac{d N_{1}}{d t}=\varepsilon_{1}\left(b_{1} R_{1}-q_{1}\right) N_{1}+\beta\left(N_{2}-N_{1}\right) \\ \frac{d N_{2}}{d t}=\varepsilon_{2}\left(b_{2} R_{2}-q_{2}\right) N_{2}+\beta\left(N_{1}-N_{2}\right) .\end{array}\right.$

\section{C.1. Positive equilibrium}

We have the following result.

Proposition 7. Let $E^{*}=\left(R_{1}^{*}, R_{2}^{*}, N_{1}^{*}, N_{2}^{*}\right)$ be a positive equilibrium of (C.1). Then $\left(N_{1}^{*}, N_{2}^{*}\right)$ is a positive equilibrium of the reduced model (14). Conversely, let $\left(N_{1}^{*}, N_{2}^{*}\right)$ be a positive equilibrium of the reduced model (14). Then $E^{*}=\left(R_{1}^{*}, R_{2}^{*}, N_{1}^{*}, N_{2}^{*}\right)$, where $R_{1}^{*}, R_{2}^{*}$ are defined by

$R_{1}^{*}=L_{1}\left(1-\frac{a_{1}}{s_{1}} N_{1}^{*}\right), \quad R_{2}^{*}=L_{2}\left(1-\frac{a_{2}}{s_{2}} N_{2}^{*}\right)$,

is a positive equilibrium of (C.1) if and only if $N_{1}^{*}<\frac{s_{1}}{a_{1}}$ and $N_{2}^{*}<\frac{s_{2}}{a_{2}}$. 
Proof. An equilibrium point $\left(R_{1}^{*}, R_{2}^{*}, N_{1}^{*}, N_{2}^{*}\right)$ of (C.1) is a solution of the set of equations

$$
\left\{\begin{array}{l}
0=\left[s_{1}\left(1-\frac{R_{1}^{*}}{L_{1}}\right)-a_{1} N_{1}^{*}\right] R_{1}^{*}, \\
0=\left[s_{2}\left(1-\frac{R_{2}^{*}}{L_{2}}\right)-a_{2} N_{2}^{*}\right] R_{2}^{*}, \\
0=\varepsilon_{1}\left(b_{1} R_{1}^{*}-q_{1}\right) N_{1}^{*}+\beta\left(N_{2}^{*}-N_{1}^{*}\right), \\
0=\varepsilon_{2}\left(b_{2} R_{2}^{*}-q_{2}\right) N_{2}^{*}+\beta\left(N_{1}^{*}-N_{2}^{*}\right) .
\end{array}\right.
$$

If this equilibrium is positive, then we must have

$$
s_{1}\left(1-\frac{R_{1}^{*}}{L_{1}}\right)-a_{1} N_{1}^{*}=0, \quad s_{2}\left(1-\frac{R_{2}^{*}}{L_{2}}\right)-a_{2} N_{2}^{*}=0 .
$$

Therefore

$$
R_{1}^{*}=L_{1}\left(1-\frac{a_{1}}{s_{1}} N_{1}^{*}\right)>0, \quad R_{2}^{*}=L_{2}\left(1-\frac{a_{2}}{s_{2}} N_{2}^{*}\right)>0 .
$$

Replacing these values in the third and fourth equations in (C.2), we get

$$
\left\{\begin{array}{l}
0=\varepsilon_{1}\left(b_{1} L_{1}-q_{1}-\frac{a_{1} b_{1}}{s_{1}} L_{1} N_{1}^{*}\right) N_{1}^{*}+\beta\left(N_{2}^{*}-N_{1}^{*}\right), \\
0=\varepsilon_{2}\left(b_{2} L_{2}-q_{2}-\frac{a_{2} b_{2}}{s_{2}} L_{2} N_{2}^{*}\right) N_{2}^{*}+\beta\left(N_{1}^{*}-N_{2}^{*}\right) .
\end{array}\right.
$$

Hence $\left(N_{1}^{*}, N_{2}^{*}\right)$ is a positive equilibrium of the reduced model (14). The reverse holds as long as the inequalities (C.3) are satisfied.

The model (C.1) was already considered by Holt (1984, his equations 6-7). In the case of resource exponential growth instead of logistic growth, he gave the condition on $\beta$ for resource persistence in both patches at equilibrium. He did not consider, however, the links between (C.1) and the reduced two-patch logistic equation (14), as we did in our study.

We will now consider the question of equilibrium resource persistence in both patches with logistic growth. More precisely, we investigate the links between the equilibrium of (C.1) and the equilibrium of the reduced model (14). Let $\left(N_{1}^{*}(\beta), N_{2}^{*}(\beta)\right)$ be a positive equilibrium of the reduced system (14). The resource abundances are positive if and only if

$N_{1}^{*}(\beta)<\frac{s_{1}}{a_{1}}, \quad N_{2}^{*}(\beta)<\frac{s_{2}}{a_{2}}$.

Recall that

$N_{1}^{*}(0)=K_{1}<\frac{s_{1}}{a_{1}}, \quad N_{2}^{*}(0)=K_{2}<\frac{s_{2}}{a_{2}}$,

where $K_{1}$ and $K_{2}$ are the carrying capacities defined by (16). Using (5) we get

$K_{1}<N_{1}^{*}(\beta)<N_{2}^{*}(\beta)<K_{2}<\frac{s_{2}}{a_{2}}$.

Hence, the condition $N_{2}^{*}(\beta)<\frac{s_{2}}{a_{2}}$ is satisfied for every $\beta>0$ and, since $N_{1}^{*}(\beta)$ is continuous with respect to $\beta$, the condition $N_{1}^{*}(\beta)<\frac{s_{1}}{a_{1}}$ is also satisfied when $\beta$ is small enough. This means that, for $\beta$ small enough, the positive equilibrium $E^{* *}$ of (C.1) defined by (18) is the same as the equilibrium $E^{*}$ considered in Proposition 7 and corresponding to the positive equilibrium $\left(N_{1}^{*}, N_{2}^{*}\right)$ of the reduced model (14). Thus, for $\beta$ small enough, we have

$N_{T}^{* *}(\beta)=N_{T}^{*}(\beta)$,

as illustrated in Fig. 5(a).
Two cases must be distinguished: $N_{1}^{*}(\beta)<\frac{s_{1}}{a_{1}}$ for all $\beta>0$, as in Fig. C.8(a), or there exists a critical value $\beta_{c}$, such that $N_{1}^{*}(\beta)<$ $\frac{s_{1}}{a_{1}}$ for $\beta<\beta_{c}$, and $N_{1}^{*}(\beta)>\frac{s_{1}}{a_{1}}$ for $\beta>\beta_{c}$, as in Fig. C.8(b). In the first case, we have $N_{T}^{* *}(\beta)=N_{T}^{*}(\beta)$ for all $\beta \geq 0$, as illustrated in Fig. 5(a). In the second case, we have $N_{T}^{* *}(\beta)=N_{T}^{*}(\beta)$ for $\beta<\beta_{c}$, as illustrated in Fig. 5(b).

Hence, when $\beta>\beta_{c}$, the equilibrium $\left(N_{1}^{*}(\beta), N_{2}^{*}(\beta)\right)$ no longer corresponds to a positive equilibrium of (C.1). Actually, the corresponding equilibrium $E^{*}$ of (C.1) described by Proposition 7, becomes negative when $\beta>\beta_{c}$, since $R_{1}^{*}(\beta)<0$.

\section{C.2. Boundary equilibrium}

Besides the equilibrium $E^{*}(\beta)=\left(R_{1}^{*}(\beta), R_{2}^{*}(\beta), N_{1}^{*}(\beta), N_{2}^{*}(\beta)\right)$ described by Proposition 7 , (C.1) can have the boundary equilibrium, as shown in the following result.

Proposition 8. The system (C.1) can have the boundary equilibrium $E^{\dagger}(\beta)=\left(0, R_{2}^{\dagger}(\beta), N_{1}^{\dagger}(\beta), N_{2}^{\dagger}(\beta)\right)$

where $R_{1}^{\dagger}(\beta)=0$ and $R_{2}^{\dagger}(\beta)>0, N_{1}^{\dagger}(\beta)>0$ and $N_{2}^{\dagger}(\beta)>0$. Let $\left(N_{1}^{*}, N_{2}^{*}\right)$ be a positive equilibrium of the reduced model (14). If $N_{1}^{*}(\beta)>\frac{s_{1}}{a_{1}}$, then we have

$N_{1}^{\dagger}(\beta)+N_{2}^{\dagger}(\beta)>N_{1}^{*}(\beta)+N_{2}^{*}(\beta)$.

Proof. The components $R_{2}^{\dagger}(\beta), N_{1}^{\dagger}(\beta)$ and $N_{2}^{\dagger}(\beta)$ of the equilibrium $E^{\dagger}(\beta)$ are the positive solutions of the set of equations

$$
\left\{\begin{array}{l}
0=s_{2}\left(1-\frac{R_{2}^{\dagger}}{L_{2}}\right)-a_{2} N_{2}^{\dagger}, \\
0=-\varepsilon_{1} q_{1} N_{1}^{\dagger}+\beta\left(N_{2}^{\dagger}-N_{1}^{\dagger}\right), \\
0=\varepsilon_{2}\left(b_{2} R_{2}^{\dagger}-q_{2}\right) N_{2}^{\dagger}+\beta\left(N_{1}^{\dagger}-N_{2}^{\dagger}\right) .
\end{array}\right.
$$

Solving the first equation for $R_{2}^{\dagger}$ yields

$R_{2}^{\dagger}=L_{2}\left(1-\frac{a_{2}}{s_{2}} N_{2}^{\dagger}\right)$.

Replacing $R_{2}^{\dagger}$ by this expression in the second and third equations of (C.5) yields

$$
\left\{\begin{array}{l}
0=-\varepsilon_{1} q_{1} N_{1}^{\dagger}+\beta\left(N_{2}^{\dagger}-N_{1}^{\dagger}\right), \\
0=\varepsilon_{2}\left(b_{2} L_{2}-q_{2}-\frac{a_{2} b_{2}}{s_{2}} L_{2} N_{2}^{\dagger}\right) N_{2}^{\dagger}+\beta\left(N_{1}^{\dagger}-N_{2}^{\dagger}\right) .
\end{array}\right.
$$

Solving the second equation for $N_{2}^{\dagger}$ yields that the equilibria are the positive intersections of the parabola $\mathscr{P}_{2}$ of equation $N_{1}=$ $P_{2}\left(N_{2}\right)$, where $P_{2}\left(N_{2}\right)$ is defined by (A.4), with $r_{2}$ and $K_{2}$ given by (15)-(16), and the straight line $\Delta$ of equation $-\varepsilon_{1} q_{1} N_{1}+\beta\left(N_{2}-\right.$ $\left.N_{1}\right)=0$ (Fig. C.9)

We want to compare the solution $\left(N_{1}^{\dagger}, N_{2}^{\dagger}\right)$ of (C.6) with the solution $\left(N_{1}^{*}, N_{2}^{*}\right)$ of (C.4). Since $N_{1}^{*}>\frac{s_{1}}{a_{1}}$, and from the first equation of (C.4), we deduce that

$-\varepsilon_{1} q_{1} N_{1}^{*}+\beta\left(N_{2}^{*}-N_{1}^{*}\right)=-\varepsilon_{1} b_{1} L_{1}\left(1-\frac{a_{1}}{s_{1}} N_{1}^{*}\right) N_{1}^{*}>0$.

Hence, the point $\left(N_{1}^{*}, N_{2}^{*}\right)$ is on the left of the straight line $\Delta$. We recall that $\left(N_{1}^{*}, N_{2}^{*}\right)$ is the positive intersection of the two parabolas $\mathscr{P}_{1}$ and $\mathscr{P}_{2}$ of equations $N_{2}=P_{1}\left(N_{1}\right)$ and $N_{1}=P_{2}\left(N_{2}\right)$, where $P_{1}\left(N_{1}\right)$ and $P_{2}\left(N_{2}\right)$ are defined by (A.4), with $r_{i}$ and $K_{i}$ given by (15)-(16). Hence we have $N_{1}^{*}<N_{1}^{\dagger}$ and $N_{2}^{*}<N_{2}^{\dagger}$, as illustrated in Fig. C.9. Therefore, we have $N_{1}^{\dagger}(\beta)+N_{2}^{\dagger}(\beta)>N_{1}^{*}(\beta)+N_{2}^{*}(\beta)$. 

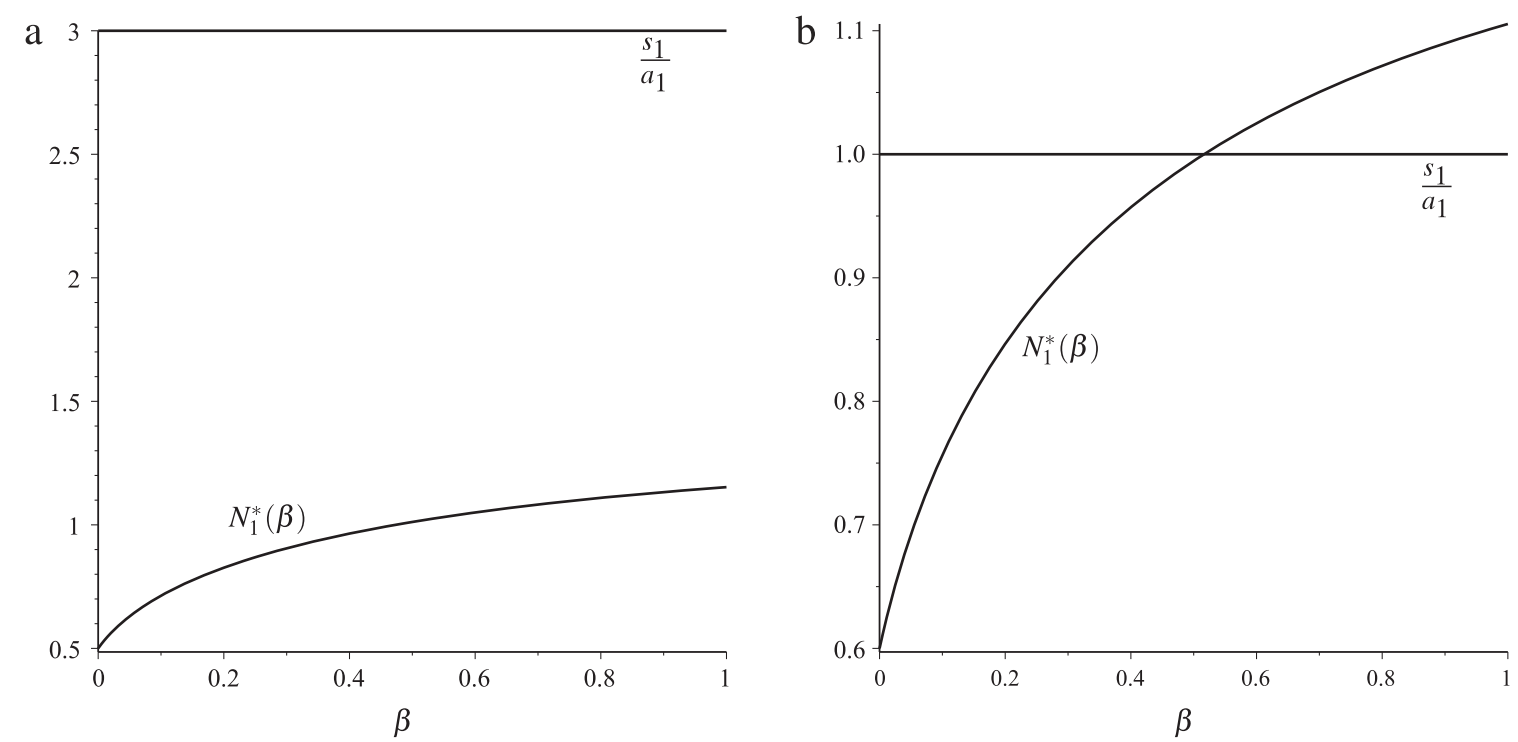

Fig. C.8. (a) $N_{1}^{*}(\beta)<\frac{s_{1}}{a_{1}}$ for all $\beta \geq 0$. (b) The critical value $\beta_{c}$ for which $N_{1}^{*}(\beta)=\frac{s_{1}}{a_{1}}$. See Fig. 5 for the plots of the corresponding total consumer population.

At $\beta=\beta_{c}$, there is a bifurcation of $E^{\dagger}(\beta)$ from $E^{*}(\beta)$. When $\beta>\beta_{c}$, we observed numerically that $E^{\dagger}(\beta)$ becomes stable and attracts all solutions. Therefore, for $\beta>\beta_{c}$, the stable equilibrium (18) of (C.1) is no longer equal to $E^{*}(\beta)$, which has become negative, but is equal to the boundary equilibrium $E^{\dagger}(\beta)$. Therefore, using Proposition 8, we have

$N_{T}^{* *}(\beta)=\left\{\begin{array}{l}N_{1}^{*}(\beta)+N_{2}^{*}(\beta)=N_{T}^{*}(\beta) \text { for } 0 \leq \beta \leq \beta_{c} \\ N_{1}^{\dagger}(\beta)+N_{2}^{\dagger}(\beta)>N_{T}^{*}(\beta) \text { for } \beta>\beta_{c}\end{array}\right.$

as illustrated in Fig. 5(b).

\section{C.3. Perfect mixing}

The behaviour of (C.1) for perfect mixing $(\beta \rightarrow \infty)$ is given by the following result.

Proposition 9. Let $\left(R_{1}(t, \beta), R_{2}(t, \beta), N_{1}(t, \beta), N_{2}(t, \beta)\right)$ be a solution of (C.1) with initial condition $\left(R_{10}, R_{20}, N_{10}, N_{20}\right)$. When $\beta \rightarrow$ $\infty$, then, with the exception of a small initial interval, $R_{1}(t, \beta)$ and $R_{2}(t, \beta)$ are approximated by $R_{1}(t)$ and $R_{2}(t)$, and $N_{1}(t, \beta)$ and $N_{2}(t, \beta)$ are both approximated by $N(t)$, where $\left(R_{1}(t), R_{2}(t), N(t)\right)$ is the solution, with initial condition $R_{10}, R_{20}$, and $N_{0}=\frac{N_{10}+N_{20}}{2}$, of the MacArthur single-patch model with two resources

$$
\left\{\begin{array}{l}
\frac{d R_{1}}{d t}=\left[s_{1}\left(1-\frac{R_{1}}{L_{1}}\right)-a_{1} N\right] R_{1} \\
\frac{d R_{2}}{d t}=\left[s_{2}\left(1-\frac{R_{2}}{L_{2}}\right)-a_{2} N\right] R_{2} \\
\frac{d N}{d t}=\varepsilon\left(c_{1} R_{1}+c_{2} R_{2}-q\right) N,
\end{array}\right.
$$

where $\varepsilon=\frac{\varepsilon_{1}+\varepsilon_{2}}{2}, c_{1}=\frac{\varepsilon_{1} b_{1}}{\varepsilon_{1}+\varepsilon_{2}}, c_{2}=\frac{\varepsilon_{2} b_{2}}{\varepsilon_{1}+\varepsilon_{2}}$, and $q=\frac{\varepsilon_{1} q_{1}+\varepsilon_{2} q_{2}}{\varepsilon_{1}+\varepsilon_{2}}$.

Proof. We use here the singular perturbation analysis outlined in Appendix A.3 to obtain the behaviour of the system as $\beta \rightarrow \infty$. Let $N=\frac{N_{1}+N_{2}}{2}$. We can rewrite (C.1) using the variables $s_{1}, s_{2}, N$ and

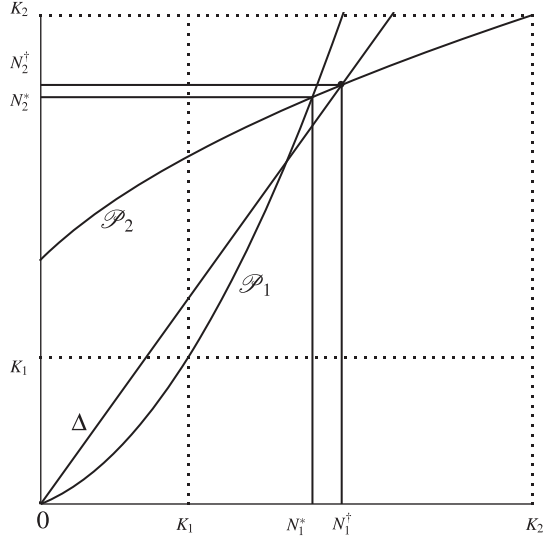

Fig. C.9. Graphical constructions of the solutions $\left(N_{1}^{\dagger}, N_{2}^{\dagger}\right)$ of (C.6) and $\left(N_{1}^{*}, N_{2}^{*}\right)$ of (C.4) showing that $N_{1}^{\dagger}>N_{1}^{*}$ and $N_{2}^{\dagger}>N_{2}^{*}$.

$N_{1}$ (using $\left.N_{2}=2 N-N_{1}\right)$ :

$\left\{\begin{array}{l}\frac{d R_{1}}{d t}=\left[s_{1}\left(1-\frac{R_{1}}{L_{1}}\right)-a_{1} N_{1}\right] R_{1} \\ \frac{d R_{2}}{d t}=\left[s_{2}\left(1-\frac{R_{2}}{L_{2}}\right)-a_{2} N_{2}\right] R_{2} \\ \frac{d N}{d t}=\frac{1}{2}\left[\varepsilon_{1}\left(b_{1} R_{1}-q_{1}\right) N_{1}+\varepsilon_{2}\left(b_{2} R_{2}-q_{2}\right)\left(2 N-N_{2}\right)\right] \\ \frac{d N_{1}}{d t}=\varepsilon_{1}\left(b_{1} R_{1}-q_{1}\right) N_{1}+2 \beta\left(N-N_{1}\right) .\end{array}\right.$

System (C.8) is a slow and fast system whose slow variables are $R_{1}, R_{2}$ and $N$, and fast variable is $N_{1}$. In the limit $\beta \rightarrow \infty$, we can replace the fast variable $N_{1}$ in the first three equations of (C.8) by its quasi-steady state approximation $N_{1}=N$ obtained from the fourth equation. We obtain

$$
\left\{\begin{array}{l}
\frac{d R_{1}}{d t}=\left[s_{1}\left(1-\frac{R_{1}}{L_{1}}\right)-a_{1} N\right] R_{1} \\
\frac{d R_{2}}{d t}=\left[s_{2}\left(1-\frac{R_{2}}{L_{2}}\right)-a_{2} N\right] R_{2} \\
\frac{d N}{d t}=\frac{1}{2}\left[\varepsilon_{1}\left(b_{1} R_{1}-q_{1}\right) N+\varepsilon_{2}\left(b_{2} R_{2}-q_{2}\right) N\right]
\end{array}\right.
$$


which is simply the MacArthur two resource-one consumer model (C.7).

In the MacArthur model (C.1), all resources are not necessarily present at equilibrium. For instance, assuming that the resources are labelled such that $\frac{s_{1}}{a_{1}}<\frac{s_{2}}{a_{2}}$ (which holds for Fig. 5(b), since in this figure one has $\frac{s_{1}}{a_{1}}=1<3=\frac{s_{2}}{a_{2}}$ ), we have the following results, which are special cases of the results in Holt (1977) obtained for the model of one consumer and $n \geq 2$ resources. (It should be noted that Holt, 1977 used the convention $\frac{s_{1}}{a_{1}}>\frac{s_{2}}{a_{2}}$, so some changes of indices occur in the following formulas.)

1. If $\varepsilon_{1} b_{1} L_{1}+\varepsilon_{2} b_{2} L_{2}>\varepsilon_{1} q_{1}+\varepsilon_{2} q_{2}$, then no resource is present at equilibrium.

2. If $\varepsilon_{1} b_{1} L_{1}+\varepsilon_{2} b_{2} L_{2} \leq \varepsilon_{1} q_{1}+\varepsilon_{2} q_{2}$, then resource $R_{2}$ is always present at equilibrium and resource $R_{1}$ is present if and only if the following condition holds:

$$
\frac{\varepsilon_{1} q_{1}+\varepsilon_{2} q_{2}}{\varepsilon_{2} b_{2} L_{2}}>1-\frac{a_{2}}{s_{2}} \frac{s_{1}}{a_{1}} \text {. }
$$

The condition (C.9) of existence of species $R_{1}$ at equilibrium is independent of its own carrying capacity $L_{1}$, yet may critically depend on $L_{2}$. This behaviour of resources sharing a common consumer is known as apparent competition.

This behaviour of the limiting model (C.7) when $\beta \rightarrow \infty$ explains why there is a critical value $\beta_{c}$ such that, for $\beta>\beta_{c}$, the resource $R_{1}$ is not present at equilibrium (see Fig. 5(b)).

From Proposition 9, we deduce the following result:

Proposition 10. If the inequality (C.9) holds, then we have

$N_{T}^{* *}(+\infty)=N_{T}^{*}(+\infty)=2 \frac{r_{1}+r_{2}}{r_{1} / K_{1}+r_{2} / K_{2}}$.

If the reverse of inequality (C.9) holds, then we have

$N_{T}^{* *}(+\infty)=2 \frac{s_{2}}{a_{2}}\left(1-\frac{\varepsilon_{1} q_{1}+\varepsilon_{2} q_{2}}{\varepsilon_{2} b_{2} L_{2}}\right)>N_{T}^{*}(+\infty)$.

Proof. If (C.9) holds, then the solutions of (C.7) converge towards the positive equilibrium $\left(R_{1}^{*}, R_{2}^{*}, N^{*}\right)$ given by

$R_{1}^{*}=L_{1}\left(1-\frac{a_{1}}{s_{1}} N^{*}\right), \quad R_{2}^{*}=L_{2}\left(1-\frac{a_{2}}{s_{2}} N^{*}\right)$,

where $N^{*}$ is the solution of the equation

$\varepsilon_{1} b_{1} L_{1}\left(1-\frac{a_{1}}{s_{1}} N^{*}\right)+\varepsilon_{2} b_{2} L_{2}\left(1-\frac{a_{2}}{s_{2}} N^{*}\right)-\varepsilon_{1} q_{1}-\varepsilon_{2} q_{2}=0$.

Hence

$N^{*}=\frac{\varepsilon_{1} b_{1} L_{1}+\varepsilon_{2} b_{2} L_{2}-\left(\varepsilon_{1} q_{1}+\varepsilon_{2} q_{2}\right)}{\varepsilon_{1} b_{1} L_{1} \frac{a_{1}}{s_{1}}+\varepsilon_{2} b_{2} L_{2} \frac{a_{2}}{s_{2}}}$.

Using Proposition 9, we see that, in the limit $\beta \rightarrow \infty$, we get $N_{1}^{* *}(+\infty)=N_{2}^{* *}(+\infty)=N^{*}$, so that $N_{T}^{* *}(+\infty)=N_{1}^{* *}(+\infty)+$
$N_{2}^{* *}(+\infty)=2 N^{*}$. Therefore, using (B.2), we see that $N_{T}^{* *}(+\infty)=$ $N_{T}^{*}(+\infty)$ and (C.10) holds.

If the reverse of inequality (C.9) holds, then $R_{1}$ is eliminated by apparent competition and the solutions of (C.7) converge towards the boundary equilibrium $\left(0, R_{2}^{*}, N^{*}\right)$ given by

$R_{2}^{*}=L_{2}\left(1-\frac{a_{2}}{s_{2}} N^{*}\right), \quad N^{*}=\frac{s_{2}}{a_{2}}\left(1-\frac{\varepsilon_{1} q_{1}+\varepsilon_{2} q_{2}}{\varepsilon_{2} b_{2} L_{2}}\right)$.

Using Proposition 9, we see that, in the limit $\beta \rightarrow \infty$, we get $N_{1}^{* *}(+\infty)=N_{2}^{* *}(+\infty)=N^{*}$, so that $N_{T}^{* *}(+\infty)=N_{1}^{* *}(+\infty)+$ $N_{2}^{* *}(+\infty)=2 N^{*}$ is given by (C.11).

\section{References}

DeAngelis, D.L., Travis, C.C., Post, W.M., 1979. Persistence and stability of seeddispersed species in a patchy environment. Theor. Popul. Biol. 16, 107-125. http://dx.doi.org/10.1016/0040-5809(79)90008-X.

DeAngelis, D.L., Zhang, B., 2014. Effects of dispersal in a non-uniform environment on population dynamics and competition: a patch model approach. Discrete Contin. Dyn. Syst. Ser. B 19, 3087-3104. http://dx.doi.org/10.3934/dcdsb.2014.19.3087.

Freedman, H.I., Waltman, D., 1977. Mathematical models of population interactions with dispersal. I. Stability of two habitats with and without a predator. SIAM J. Appl. Math. 32, 631-648. http://dx.doi.org/10.1137/0132052.

Gabriel, J.P., Saucy, F., Bersier, L.F., 2005. Paradoxes in the logistic equation? Ecol Modell. 185, 147-151. http://dx.doi.org/10.1016/j.ecolmodel.2004.10.009.

Ginzburg, L.R., 1992. Evolutionary consequences of basic growth equations. Trends Ecol. Evol. 7, 133-133. http://dx.doi.org/10.1016/0169-5347(92)90149-6.

Hanski, I., 1999. Metapopulation Ecology. Oxford University Press.

Holt, R.D., 1977. Predation, apparent competition, and the structure of prey communities. Theor. Popul. Biol. 12, 197-229. http://dx.doi.org/10.1016/00405809(77)90042-9.

Holt, R.D., 1984. Spatial heterogeneity, indirect interactions, and the coexistence of prey species. Am. Nat. 124, 377-406. Stable URL: http://www.jstor.org/stable/2461465.

Holt, R.D., 1985. Population dynamics in two-patch environments: Some anomalous consequences of an optimal habitat distribution. Theor. Popul. Biol. 28, 181-208. http://dx.doi.org/10.1016/0040-5809(85)90027-9.

Kuno, E., 1991. Some strange properties of the logistic equation defined with $r$ and $K$ : inherent defects or artifacts? Res. Popul. Ecol. 33, 33-39. http://dx.doi.org/10.1007/BF02514572.

Lobry, C., Sari, T., Touhami, S., 1998. On Tykhonov's theorem for convergence of solutions of slow and fast systems. Electron. J. Differential Equations 1998 (19), $1-22$.

MacArthur, R.H., 1969. Species packing and what interspecies competition minimizes. Proc. Natl. Acad. Sci. 64, 1369-1371.

MacArthur, R.H., 1970. Species packing and competitive equilibrium for many species. Theor. Popul. Biol. 1, 1-11. http://dx.doi.org/10.1016/00405809(70)90039-0.

Mallet, J., 2012. The struggle for existence: how the notion of carrying capacity, $K$, obscures the links between demography, Darwinian evolution, and speciation. Evol. Ecol. Res. 14, 627-665.

Pearl, R., 1927. The growth of populations. Q. Rev. Biol. 2, 532-548. Stable URL: http://www.jstor.org/stable/2808218.

Poggiale, J.-C., Auger, P., Nérini, D., Manté, C., Gilbert, F., 2005. Global production increased by spatial heterogeneity in a population dynamics model. Acta Biotheor. 53, 359-370.

Reynolds, S.A., Brassil, C.E., 2013. When can a single-species, density-dependent model capture the dynamics of a consumer-resource system? J. Theoret. Biol. 339, 70-83. http://dx.doi.org/10.1016/j.jtbi.2013.08.025.

Tikhonov, A.N., 1952. Systems of differential equations containing small parameters in the derivatives. Mat. Sb. (N.S.) 31 (73), 575-586. (in Russian).

Verhulst, P.F., 1838. Notice sur la loi que la population suit dans son accroissement. Corresp. Math. Phys. 10, 113-121

Wasow, W.R., 1976. Asymptotic Expansions for Ordinary Differential Equations. Robert E. Krieger Publishing Company, Huntington, NY. 